
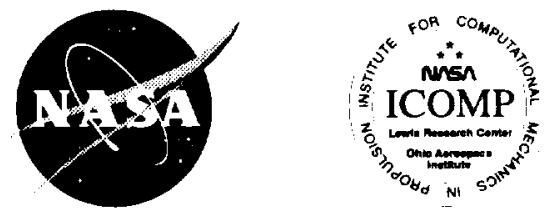

\title{
Preconditioning for Numerical Simulation of Low Mach Number Three-Dimensional Viscous Turbomachinery Flows
}

Daniel L. Tweedt and Rodrick V. Chima

Lewis Research Center, Cleveland, Ohio

Eli Turkel

Institute for Computational Mechanics in Propulsion, Cleveland, Ohio and Tel-Aviv University, Tel-Aviv, Israel

Prepared for the 28th Fluid Dynamics Conference sponsored by the American Institute of Aeronautics and Astronautics Snowmass, Colorado, June 29-July 2, 1997

National Aeronautics and Space Administration

Lewis Research Center 
Available from

NASA Center for Aerospace Information 800 Elkridge Landing Road

Lynthicum, MD 21090-2934

Price Code: A03
National Technical Information Service 5287 Port Royal Road Springfield, VA 22100 Price Code: A03 


\title{
Preconditioning for Numerical Simulation of Low Mach Number Three-Dimensional Viscous Turbomachinery Flows
}

\author{
Daniel L. Tweedt ${ }^{\dagger}$ and Rodrick V. Chima ${ }^{\ddagger}$ \\ NASA Lewis Research Center \\ Cleveland, Ohio 44135 \\ Eli Turkel ${ }^{\S}$ \\ School of Mathematical Sciences \\ Tel-Aviv University, Tel-Aviv, Israel \\ and ICOMP, NASA Lewis Research Center
}

\begin{abstract}
A preconditioning scheme has been implemented into a three-dimensional viscous computational fluid dynamics code for turbomachine blade rows. The preconditioning allows the code, originally developed for simulating compressible flow fields, to be applied to nearly-incompressible, low Mach number flows. A brief description is given of the compressible Navier-Stokes equations for a rotating coordinate system, along with the preconditioning method employed. Details about the conservative formulation of artificial dissipation are provided, and different artificial dissipation schemes are discussed and compared. The preconditioned code was applied to a well-documented case involving the NASA large low-speed centrifugal compressor for which detailed experimental data are available for comparison. Performance and flow field data are compared for the near-design operating point of the compressor, with generally good agreement between computation and experiment. Further, significant differences between computational results for the different numerical implementations, revealing different levels of solution accuracy, are discussed.
\end{abstract}

\footnotetext{
${ }^{\dagger}$ Aerospace Engineer

${ }^{\ddagger}$ Aerospace Engineer, Associate Fellow AIAA

${ }^{\S}$ Professor, Department of Mathematics, Senior Member AIAA
}

\section{Introduction}

The general engineering discipline of turbomachinery fluid dynamics involves a wide spectrum of practical devices and machines, many of which involve incompressible or nearly incompressible fluid flows. A list of such devices would include, among others, pumps, hydraulic turbines, propellers, low-speed fans and blowers, and lowspeed experimental test rigs. Despite this abundance of incompressible-flow turbomachinery, and other incompressible flows in general, it is an interesting historical facet of computational fluid dynamics (CFD) that a great many computer codes for fluid dynamic simulation have been developed based on numerical algorithms intended only for compressible flows. One reason for this is that most state-of-the-art fluids engineering is being performed in the aeropropulsion industry where the fluid dynamics is mostly compressible, and where new technology is worth the high cost of expensive research and development, including CFD code development.

It is well known that most compressible-flow CFD codes will not converge to an acceptable flow solution when the flow field Mach numbers become too low. Typically this occurs somewhere below about Mach number 0.1 where the gas becomes virtually incompressible; that is, the fluid density becomes nearly constant with changes in flow velocity. Within the past several years, however, methods for altering the compressible-flow numerical algorithm to allow convergence at very low Mach numbers have increasingly appeared in the literature [1-10]. These so-called preconditioning methods alter the eigenvalues of the system of compressible-flow equations so as to reduce, at low Mach numbers, the large disparity between the acoustic and convective wave speeds. Although it is possible to avoid this problem altogether by developing the numerical algorithm to solve the incompressible flow 
equations, the preconditioned compressible codes have several advantages and/or benefits, not the least of which is that many compressible-flow codes already exist for various applications. It is relatively easy to add a preconditioning scheme to an already existing code. Other advantages include code versatility and the capability to directly simulate flow fields involving both compressible and incompressible flow regions.

In this paper, a three-dimensional viscous CFD code originally developed for the simulation of the compressible flow field within a turbomachine blade row is considered. The code, designated RVC3D, solves a thinlayer formulation of the Reynolds-averaged three-dimensional Navier-Stokes equations [11, 12], and uses the Baldwin-Lomax algebraic turbulence model [13] to simulate the effects of boundary layer turbulence. Preconditioning has been incorporated into the code by implementing the work of Turkel [3, 4], Radespiel and Turkel [7], Radespiel et al. [6], and Turkel et al. [10]. The paper describes the numerical method, including the preconditioning scheme, different artificial dissipation schemes, and some problems encountered. Computed results for a centrifugal compressor impeller are then presented and compared to detailed experimental data.

\section{Governing Equations}

The governing equations which are numerically integrated using the RVC3D code are summarized below. Only the final thin-layer formulation of the viscous compressible equations as transformed to a generalized bodyfitted coordinate system are presented here since a more comprehensive description can be found in the references for RVC3D [11, 12].

The Navier-Stokes equations are written for a Carte$\operatorname{sian}(x, y, z)$ coordinate system rotating with angular velocity $\Omega$ about the $x$-axis. The Cartesian equations have been mapped to a body-fitted $(\xi, \eta, \zeta)$ coordinate system, simplified using the thin-layer approximation, and nondimensionalized by arbitrary reference quantities $\rho_{0}, a_{0}$, and $\mu_{0}$. Note that the $\xi$-coordinate direction is roughly parallel to the blade surface and wraps around it, while the $\eta$ direction is almost normal to the blade surface and the $\zeta$ direction runs along the blade span. The resulting equations can be summarized as follows:

$$
\partial_{t} \hat{q}+\partial_{\xi} \hat{E}+\partial_{\eta} \hat{F}+\partial_{\zeta} \hat{G}-R e^{-1}\left(\partial_{\eta} \hat{F_{V}}+\partial_{\zeta} \hat{G_{V}}\right)=\hat{H}
$$

where

$$
\hat{q}=J^{-1} q=J^{-1}[\rho, \rho u, \rho v, \rho w, e]^{T}
$$

NASA TM-1 13120

$$
\begin{aligned}
\hat{E} & =J^{-1}\left[\begin{array}{l}
\rho U^{\prime} \\
\rho u U^{\prime}+\xi_{x} p \\
\rho v U^{\prime}+\xi_{y} p \\
\rho w U^{\prime}+\xi_{z} p \\
e U^{\prime}+p U
\end{array}\right] \\
\hat{G}=J^{-1}\left[\begin{array}{l}
\rho W^{\prime} \\
\rho u W^{\prime}+\zeta_{x} p \\
\rho v W^{\prime}+\zeta_{y} p \\
\rho w W^{\prime}+\zeta_{z} p \\
e W^{\prime}+p W
\end{array}\right] & {\left[\begin{array}{l}
\rho V^{\prime} \\
\rho u V^{\prime}+\eta_{x} p \\
\rho v V^{\prime}+\eta_{y} p \\
\rho w V^{\prime}+\eta_{z} p \\
e V^{\prime}+p V
\end{array}\right] }
\end{aligned}
$$

Details of the viscous flux vectors $\hat{F}_{V}$ and $\hat{G}_{V}$ are given in References 11 and 12 . The absolute velocity components $u, v$ and $w$ point in the $x, y$, and $z$ coordinate directions, respectively, and the relative contravariant velocity components are given by

$$
\begin{aligned}
& U^{\prime}=\xi_{x} u+\xi_{y} v^{\prime}+\xi_{z} w^{\prime} \\
& V^{\prime}=\eta_{x} u+\eta_{y} v^{\prime}+\eta_{z} w^{\prime} \\
& W^{\prime}=\zeta_{x} u+\zeta_{y} v^{\prime}+\zeta_{z} w^{\prime}
\end{aligned}
$$

where the relative velocity components are:

$$
\begin{aligned}
u^{\prime} & =u \\
v^{\prime} & =v-\Omega z \\
w^{\prime} & =w+\Omega y
\end{aligned}
$$

Assuming an ideal gas with constant specific heats, then $\gamma\left(=c_{p} / c_{v}\right)$ is constant, and the energy and static pressure are given by

$$
\begin{aligned}
& e=\rho\left[\frac{a^{2}}{\gamma(\gamma-1)}+\frac{1}{2}\left(u^{2}+v^{2}+w^{2}\right)\right] \\
& p=(\gamma-1)\left[e-\frac{1}{2} \rho\left(u^{2}+v^{2}+w^{2}\right)\right.
\end{aligned}
$$

where the sonic velocity $a$ is related to static pressure and density by the equation of state:

$$
a^{2}=\frac{\gamma p}{\rho}
$$

The metric terms are defined by the following relations: 


$$
\begin{aligned}
& {\left[\begin{array}{ll}
\xi_{x} \eta_{x} \zeta_{x} \\
\xi_{y} \eta_{y} \zeta_{y} \\
\xi_{z} \eta_{z} \zeta_{z}
\end{array}\right]=} \\
& \quad\left[\begin{array}{l}
y_{\eta} z_{\zeta}-y_{\zeta} z_{\eta} y_{\zeta} z_{\xi}-y_{\xi} z_{\zeta} y_{\xi} z_{\eta}-y_{\eta} z_{\xi} \\
x_{\zeta} z_{\eta}-x_{\eta} z_{\zeta} x_{\xi} z_{\zeta}-x_{\zeta} z_{\xi} x_{\eta} z_{\xi}-x_{\xi} z_{\eta} \\
x_{\eta} y_{\zeta}-x_{\zeta} y_{\eta} x_{\zeta} y_{\xi}-x_{\xi} y_{\zeta} x_{\xi} y_{\eta}-x_{\eta} y_{\xi}
\end{array}\right]
\end{aligned}
$$

where the Jacobian $J$ and its inverse $J^{-1}$ are

$$
\begin{aligned}
J^{-1}= & x_{\xi} y_{\eta} z_{\zeta}+x_{\zeta} y_{\xi} z_{\eta}+x_{\eta} y_{\zeta} z_{\xi} \\
& -x_{\xi} y_{\zeta} z_{\eta}-x_{\eta} y_{\xi} z_{\zeta}-x_{\zeta} y_{\eta} z_{\xi}
\end{aligned}
$$

The inlet (inflow) boundary condition for the above system of equations involves specification of the spanwise distributions of total pressure, total temperature, and absolute circumferential velocity component $v_{\theta}$. The (nonpreconditioned) upstream-running Riemann-invariant based on the meridional velocity component is extrapolated upstream from the interior to the inlet boundary.

At the exit boundary four of the five conservation variables, namely $\rho, \rho u, \rho v$, and $\rho w$, are extrapolated downstream to the boundary, and the static pressure is specified at the inner (hub) boundary and integrated in the spanwise direction using simple radial equilibrium:

$$
\frac{d p}{d r}=\frac{\bar{\rho} \bar{v}_{\theta}^{2}}{r}
$$

where $r$ is local radius and the overbar denotes a circumferentially-averaged quantity. The resulting local spanwise static pressures are then used either as constants (circumferentially-invariant exit static pressures), or as nominal values for calculating circumferential pressure distributions using a method described by Giles [14].

Circumferentially-periodic boundaries between the blades are solved like interior points using a dummy grid line outside of the domain. At blade and endwall surfaces the no-slip, adiabatic wall boundary condition is used (for viscous simulations).

\section{Numerical Method}

The governing equations are discretized using a nodecentered finite-difference scheme, with second-order central differences used throughout. The multistage RungeKutta scheme developed by Jameson, Schmidt, and Turkel [15] is used to advance the flow equations in time from an initial guess to a steady state. With the exception of the 5 - stage scheme, the physical and artificial dissipation terms are calculated only at the first stage and then held constant for subsequent stages. Further details of the Runge-Kutta solution scheme can be found in References 11 and 12 .

The time step limit resulting from a three-dimensional linear stability analysis can be expressed as follows:

$$
\Delta t \leq \frac{\Lambda^{*}}{\Delta t_{\xi}^{-1}+\Delta t_{\eta}^{-1}+\Delta t_{\zeta}^{-1}+\Delta t_{V}^{-1}}
$$

where $\Lambda^{*}$ is the maximum Courant number for the particular multistage scheme. The inverse one-dimensional time steps for each grid direction are equal to the largest local eigenvalue magnitudes (for the inviscid equations without preconditioning):

$$
\begin{aligned}
& \Delta t_{\xi}^{-1}=\lambda_{\xi}=\left|U^{\prime}\right|+a \sigma_{\xi} \\
& \Delta t_{\eta}^{-1}=\lambda_{\eta}=\left|\mathrm{V}^{\prime}\right|+a \sigma_{\eta} \\
& \Delta t_{\zeta}^{-1}=\lambda_{\zeta}=\left|\mathrm{W}^{\prime}\right|+a \sigma_{\zeta}
\end{aligned}
$$

where

$$
\begin{aligned}
& \left|U^{\prime}\right|=\left|\xi_{x} u\right|+\left|\xi_{y} v^{\prime}\right|+\left|\xi_{z} w^{\prime}\right| \\
& \left|\mathrm{V}^{\prime}\right|=\left|\eta_{x} u\right|+\left|\eta_{y} v^{\prime}\right|+\left|\eta_{z} w^{\prime}\right| \\
& \left|\mathrm{W}^{\prime}\right|=\left|\zeta_{x} u\right|+\left|\zeta_{y} v^{\prime}\right|+\left|\zeta_{z} w^{\prime}\right|
\end{aligned}
$$

and

$$
\begin{aligned}
& \sigma_{\xi}=\sqrt{\xi_{x}^{2}+\xi_{y}^{2}+\xi_{z}^{2}} \\
& \sigma_{\eta}=\sqrt{\eta_{x}^{2}+\eta_{y}^{2}+\eta_{z}^{2}} \\
& \sigma_{\zeta}=\sqrt{\zeta_{x}^{2}+\zeta_{y}^{2}+\zeta_{z}^{2}}
\end{aligned}
$$

The viscous time-step [16] contribution $\Delta t_{V}^{-1}$ is given by

$$
\Delta t_{V}^{-1}=k_{t} \frac{\gamma \mu}{\rho \operatorname{Pr}}\left(\sigma_{\xi}^{2}+\sigma_{\eta}^{2}+\sigma_{\zeta}^{2}\right)
$$

where the constant $k_{t}$ is assigned a value of 4.0 .

To accelerate convergence to a steady state, the maximum permissible time step at each grid point is used, giving a constant Courant number everywhere. To further accelerate convergence, implicit residual smoothing [17] is used to allow a time step typically two to three times larger than the stability limit given by Equation 10. Eigenvalue scaling [18] of the residual smoothing coefficients, and of the artificial dissipation where applicable, is employed and greatly increases the accuracy and robustness of the numerical scheme. A blending function nearly 
the same as one proposed by Kunz and Lakshminarayana [19] is used to blend the eigenvalues from all three directions.

\section{Preconditioning}

The implementation of preconditioning into the RVC3D code is based on the work of Turkel [3, 4], Radespiel and Turkel [7], Radespiel et al. [6], and Turkel et al. [10], where generalized preconditioning schemes have been developed and summarized. The most recent publications by the above authors present a generalization of the preconditioners originally introduced by Turkel $[3,4]$, and by Choi and Merkle [5], and they also present some further developments since then. In contrast to "standard" numerical schemes using a pseudo-compressibility approach, the preconditioners they describe not only accelerate the convergence of the numerical solution to a steady state, but also change the steady-state solution because of the choice of artificial viscosity terms. As discussed in Reference 2, the standard pseudo-compressibility schemes do not converge to the solution of the incompressible equations as the Mach number approaches zero. With proper preconditioning, however, the numerical scheme does behave appropriately at low Mach numbers. The recent publications also show that preconditioning can be combined with well-known convergence acceleration techniques such as residual smoothing and multigrid.

Only one of several possible schemes has been incorporated into the RVC3D code, which is a scheme closely resembling the preconditioner of Choi and Merkel [5]. The scheme as implemented has been formulated to maintain a conservative form of artificial dissipation.

For convenience, Equation 1 is rewritten in a terse finite-difference form:

$$
\Delta \hat{q}=-\Delta t\left[R_{I}-\left(R_{V}+D\right)\right]
$$

where $R_{I}$ is the inviscid residual including source term, $R_{V}$ is the viscous residual, and $D$ is the artificial dissipation term. The artificial dissipation term can be expanded as follows:

$$
D=\left(\Delta_{\xi} \nabla_{\xi}+\Delta_{\eta} \nabla_{\eta}+\Delta_{\zeta} \nabla_{\zeta}\right) \hat{q}
$$

where each coordinate-direction appears explicitly, and $\nabla_{\xi}, \nabla_{\eta}$, and $\nabla_{\zeta}$, are operators defined later which depend on the type of artificial dissipation scheme. Denoting the preconditioning matrix by $\Gamma$, the preconditioned form of Equation 15 can be expressed as follows:

$$
\begin{aligned}
\Delta \hat{\omega}= & -\Gamma \Delta t\left[R_{I}-\left\{R_{V}+\right.\right. \\
& \left.\left.\left(\Delta_{\xi} \Gamma^{-1} \nabla_{\xi}+\Delta_{\eta} \Gamma^{-1} \nabla_{\eta}+\Delta_{\zeta} \Gamma^{-1} \nabla_{\zeta}\right) \hat{\omega}\right\}\right]
\end{aligned}
$$

where the solution vector $\hat{q}$ has been replaced by $\hat{\omega}$ :

$$
\hat{\omega}=J^{-1} \omega=J^{-1}[p, u, v, w, h]^{T}
$$

and $h$ is static enthalpy:

$$
h=a^{2} /(\gamma-1)
$$

The preconditioning matrix $\Gamma$ and its inverse $\Gamma^{-1}$ are given by

$$
\Gamma=\frac{1}{\rho}\left[\begin{array}{ccccc}
\beta^{2} \rho & 0 & 0 & 0 & 0 \\
-u & 1 & 0 & 0 & 0 \\
-v & 0 & 1 & 0 & 0 \\
-w & 0 & 0 & 1 & 0 \\
\left(\beta^{2}+\frac{q^{2}}{2}-\frac{a^{2}}{\gamma-1}\right) & -u & -v & -w & 1
\end{array}\right]
$$

$$
\Gamma^{-1}=\left[\begin{array}{ccccc}
1 / \beta^{2} & 0 & 0 & 0 & 0 \\
u / \beta^{2} & \rho & 0 & 0 & 0 \\
v / \beta^{2} & 0 & \rho & 0 & 0 \\
w / \beta^{2} & 0 & 0 & \rho & 0 \\
\left(\frac{h}{\beta^{2}}+\frac{q^{2}}{2 \beta^{2}}-1\right) & \rho u & \rho v & \rho w & \rho
\end{array}\right]
$$

where $q^{2}=u^{2}+v^{2}+w^{2}$, and for the rotating coordinate system

$$
\begin{aligned}
\beta^{2} & =\min \left[\max \left(q^{\prime 2}, k_{p} q_{r e f}^{\prime 2}\right), a^{2}\right] \\
q^{\prime 2} & =u^{2}+v^{\prime 2}+w^{\prime 2}
\end{aligned}
$$

The parameters $k_{p}$ and $q_{r e f}^{\prime}$ are specified constants, with $k_{p}$ typically between 0.1 and 0.3 for viscous simulations, and $q_{\text {ref }}^{\prime}$ being a nominal reference velocity in the relative frame-of-reference.

The largest eigenvalues for the resulting preconditioned (inviscid) equations are the following for each coordinate direction: 


$$
\begin{aligned}
& \lambda_{\xi}=\kappa\left|U^{\prime}\right|+\sqrt{\kappa^{2}\left|U^{\prime}\right|^{2}+\beta^{2}\left(\sigma_{\xi}^{2}-\frac{\left|U^{\prime}\right|^{2}}{a^{2}}\right)} \\
& \lambda_{\eta}=\kappa\left|V^{\prime}\right|+\sqrt{\kappa^{2}\left|V^{\prime}\right|^{2}+\beta^{2}\left(\sigma_{\eta}^{2}-\frac{V^{\prime 2}}{a^{2}}\right)} \\
& \lambda_{\zeta}=\kappa\left|W^{\prime}\right|+\sqrt{\kappa^{2}\left|W^{\prime}\right|^{2}+\beta^{2}\left(\sigma_{\zeta}^{2}-\frac{\left|W^{\prime}\right|^{2}}{a^{2}}\right)}
\end{aligned}
$$

where

$$
\kappa=\frac{1}{2}\left(1+\frac{\beta^{2}}{a^{2}}\right)
$$

and the relative contravariant velocity magnitudes $\left|U^{\prime}\right|$, $\left|V^{\prime}\right|$, and $\left|W^{\prime}\right|$, are obtained from Equation 12.

The above eigenvalues are used to determine the time step and to directionally scale the implicit residual smoothing and the artificial dissipation in the same way as for the unpreconditioned scheme.

Theoretical considerations suggest that modifications to the boundary condition formulations might be needed in conjunction with the preconditioning scheme $[6,10]$. Good numerical convergence behavior was observed for all cases investigated, however, without such modifications. Therefore no changes were made to any of the boundary condition formulations.

\section{Artificial Dissipation Models}

Two basic artificial dissipation models are examined for use with the preconditioning scheme at low Mach numbers. The first model is that introduced by Jameson, Schmidt, and Turkel [15], and will be referred to as the baseline scheme. This scheme uses blended first- and third-order dissipation terms and is the one originally used in the RVC3D code as documented in the references [11, 12]. The second model involves scaling the artificial dissipation by the local convective eigenvalue. At low Mach numbers (below Mach 0.5) this scheme is identical to the Convective Upwind Split Pressure (CUSP) scheme introduced fairly recently by Jameson [20], and subsequently modified by Tatsumi, Martinelli, and Jameson [21, 22]. The Symmetric LImited Positive (SLIP) flux limiter introduced by Jameson [20] is used in connection with this scheme, which for brevity will be referred to in this paper simply as the SLIP scheme. A variation of the SLIP scheme is also introduced, the ASymmetric LImited Positive (ASLIP) scheme. For the low Mach number levels considered, the ASLIP scheme (convective-eigenvalue scaling with ASLIP flux limiter) appears to work slightly better than the SLIP scheme (convective-eigenvalue scaling with SLIP flux limiter).

The baseline and the SLIP/ASLIP schemes can be described using the dissipation operators $\Delta_{\xi} \nabla_{\xi}, \Delta_{\eta} \nabla_{\eta}$, and $\Delta_{\zeta} \nabla_{\zeta}$ in Equation 16 as the starting point. Since each directional operator is similar to the others, only the $\xi$ direction need be considered. Also, note that $q$ everywhere below is replaced by $\omega$ when preconditioning is applied.

\section{Baseline Scheme}

In the baseline scheme, the dissipation term $\Delta_{\xi} \nabla_{\xi} \hat{q}$ is finite-differenced according to

$$
\begin{aligned}
\Delta_{\xi} \nabla_{\xi} \hat{q}= & \left(J^{-1} C_{\xi}\right)_{i+1 / 2} \delta_{i+1 / 2}- \\
& \left(J^{-1} C_{\xi}\right)_{i-1 / 2} \delta_{i-1 / 2}
\end{aligned}
$$

where the $i$-indexing corresponds to the $\xi$-direction and $C_{\xi}$ is a coefficient obtained from blended eigenvalues [19]:

$$
C_{\xi}=\lambda_{\xi} \sqrt{1+\frac{\lambda_{\eta}+\lambda_{\zeta}}{\lambda_{\xi}}}
$$

The dissipative flux vector $\delta_{i+1 / 2}$ is given by

$$
\begin{aligned}
\delta_{i+1 / 2}= & V_{2} \Delta q_{i+1 / 2} \\
& V_{4}\left(\Delta q_{i+3 / 2}-2 \Delta q_{i+1 / 2}+\Delta q_{i-1 / 2}\right)
\end{aligned}
$$

where $\Delta q_{i+1 / 2}=q_{i+1}-q_{i}$ and the coefficients $V_{2}$ and $V_{4}$ are the following:

$$
\begin{aligned}
& v_{2}=\mu_{2} \max \left(v_{i-1}, v_{i}, v_{i+1}, v_{i+2}\right) \\
& v_{4}=\max \left(0, \mu_{4}-v_{2}\right)
\end{aligned}
$$

with

$$
v_{i}=\frac{\left|p_{i+1}-2 p_{i}+p_{i-1}\right|}{\min \left(p_{n},\left|p_{i+1}+2 p_{i}+p_{i-1}\right|\right)}
$$

The constant $\mu_{2}$ scales a first-order artificial viscosity that is switched on at shocks detected by Equation 29. The denominator in Equation 29 is normally constant at the pressure $p_{n}$, a fixed low pressure at either the grid inlet or exit, making the operator roughly symmetric across shocks. The more commonly applied term $\left|p_{i+1}+2 p_{i}+p_{i-1}\right|$ is included to switch on the seconddifference dissipation when the pressure becomes very 
small, usually due to numerical problems. The constant $\mu_{4}$ scales a uniform third-order artificial viscosity that is switched off at shocks by $V_{2}$. Typically, values for $\mu_{2}$ and $\mu_{4}$ are $1 / 4$ and $1 / 32$, respectively.

\section{SLIP/ASLIP Scheme}

In the SLIP/ASLIP scheme, the dissipation term $\Delta_{\xi} \nabla_{\xi} \hat{q}$ is finite-differenced according to

$$
\begin{aligned}
\Delta_{\xi} \nabla_{\xi} \hat{q}= & \left(J^{-1} \sigma_{\xi}\right)_{i+1} \delta_{i+1 / 2} \\
& \left(J^{-1} \sigma_{\xi}\right)_{i-1} \delta_{i-1 / 2}
\end{aligned}
$$

where $\sigma_{\xi}$ is given by Equation 13, and the dissipative flux vector $\delta_{i+1 / 2}$ is given by

$$
\delta_{i+1 / 2}=\frac{1}{2} \alpha a_{i+1 / 2}\left(q_{R}-q_{L}\right)
$$

The subscripts $R$ and $L$ denote right and left states, respectively, for the state vector $q$. The left and right states are determined by either the SLIP or the ASLIP flux limiter, as described later. The coefficient $\alpha$ is given by

$$
\alpha=\varsigma^{+}\left\{\begin{array}{cl}
\left|M_{\xi}^{\prime}\right| & \left|M_{\xi}^{\prime}\right| \geq \zeta \varepsilon_{0}^{-} \\
\frac{1}{2}\left(\zeta \varepsilon_{0}+\frac{M_{\xi}^{\prime 2}}{\zeta \varepsilon_{0}}\right) & \left|M_{\xi}^{\prime}\right|<\zeta \varepsilon_{0}^{-}
\end{array}\right.
$$

where the relative contravariant Mach number $M_{\xi}^{\prime}$ is

$$
M_{\xi}^{\prime}=\frac{\overline{U^{\prime}}}{\left(a \sigma_{\xi}\right)_{i+1 / 2}}=\frac{U_{R}^{\prime}+U_{L}^{\prime}}{2\left(a \sigma_{\xi}\right)_{i+1 / 2}}
$$

When preconditioning is applied, $q_{R}-q_{L}$ is replaced by $\Gamma_{i+1 / 2}^{-1}\left(\omega_{R}-\omega_{L}\right)$ in Equation 31 .

The parameters $\varsigma^{+}$and $\varsigma^{-}$rescale the artificial dissipation near the wall for viscous flows, for reasons discussed in Reference 23. For convenience and computational efficiency, the coefficients obtained from blended eigenvalues (see Equation 26) are used:

$$
\begin{aligned}
\varsigma^{+} & =\max \left(1,\left[1-2\left|M_{\xi}^{\prime}\right|\right] \varsigma_{\xi}\right) \\
\varsigma^{-} & =\min \left(1, \varsigma_{\xi}^{2}\right) \\
\varsigma_{\xi} & =\frac{C_{\eta}+C_{\zeta}}{C_{\xi}}
\end{aligned}
$$

The parameter $\varsigma^{+}$as given above is used only in the $\xi$ direction, with $\varsigma^{+}$being set identically to one for the $\eta$ and $\zeta$-directions (where physical viscosity terms exist). The parameters $\varepsilon_{0}$ is defined by

$$
\varepsilon_{0}=k_{v} M_{r e f}^{\prime}
$$

where $k_{v}$ and $M^{\prime}{ }_{\text {ref }}$ are specified constants, with $k_{v}$ typically given a value of 0.05 or less for viscous simulations, and $M^{\prime}{ }_{r e f}$ being a nominal reference Mach number in the relative frame-of-reference.

As alluded to earlier, either the SLIP or ASLIP flux limiter is used to determine the left and right states. This is done by extrapolation from nearby data, subject to a limiter to preserve monotonicity. This approach is similar to van Leer's MUSCL scheme [24] and provides firstorder dissipation near "discontinuities", that is, nonsmooth regions, and third-order dissipation in smooth regions of the flow field.

From the work of Swanson, Radespiel, and Turkel [23], and considering the right and left states in the dissipative flux vector $\delta_{i+1 / 2}$, a slightly generalized form of the SLIP limiter can be written as follows:

$$
\begin{aligned}
& q_{L}=q_{i}+\frac{1}{2} L\left(\Delta q_{i+3 / 2}, \Delta q_{i+1 / 2}, \Delta q_{i-1 / 2}\right) \\
& q_{R}=q_{i+1}-\frac{1}{2} L\left(\Delta q_{i+3 / 2}, \Delta q_{i+1 / 2}, \Delta q_{i-1 / 2}\right)
\end{aligned}
$$

where $\Delta q_{i+1 / 2}=q_{i+1}-q_{i}$, and the operator $L$ is defined as

$$
\begin{aligned}
L\left(\Delta q^{+}, \Delta q, \Delta q^{-}\right) & =s\left(\Delta q^{+}, \Delta q^{-}\right) \\
& {\left[\left(1-\mu_{4}\right) \Delta q+\mu_{4} \frac{\Delta q^{+}+\Delta q^{-}}{2}\right] }
\end{aligned}
$$

with the switch $S$ as

$$
s\left(\Delta q^{+}, \Delta q^{-}\right)=1-{\frac{\Delta q^{+}-\Delta q^{-}}{\left|\Delta q^{+}\right|+\left|\Delta q^{-}\right|+\varepsilon}}^{\chi}
$$


The exponent $\chi$ is a positive number given a value of 2.0 in the RVC3D code. The constant $\mu_{4}$ scales the third-order dissipation such that in smooth regions of the flow $(S \approx 1)$ :

$$
q_{R}-q_{L} \approx-\frac{\mu_{4}}{2} \Delta^{3} q_{i+1 / 2}
$$

Typically $\mu_{4}$ is specified to have a value of 1.0 . Note that whenever $\Delta q^{+}$and $\Delta q^{-}$have the opposite sign then $S \approx 0$, and where the flow is smooth then $S \approx 1$. The parameter $\varepsilon$ in Equation 38 is a threshold to prevent the denominator from getting close to zero when both $\Delta q^{+}$ and $\Delta q^{-}$are small.

The ASLIP limiter is similar to the SLIP limiter except that the operator $L$ is split into two parts:

$$
L\left(\Delta q^{+}, \Delta q, \Delta q^{-}\right)=\frac{1}{2} L^{+}\left(\Delta q^{+}, \Delta q\right)+\frac{1}{2} L^{-}\left(\Delta q^{-}, \Delta q\right)
$$

where

$$
\begin{aligned}
L^{+}\left(\Delta q^{+}, \Delta q\right)= & s\left(\Delta q^{+}, \Delta q\right) \\
& {\left[\left(1-2 \mu_{4}\right) \Delta q+\mu_{4}\left(\Delta q^{+}+\Delta q\right)\right] } \\
L^{-}\left(\Delta q^{-}, \Delta q\right)= & S\left(\Delta q^{-}, \Delta q\right) \\
& {\left[\left(1-2 \mu_{4}\right) \Delta q+\mu_{4}\left(\Delta q^{-}+\Delta q\right)\right] }
\end{aligned}
$$

The switch $S$ is defined the same as above. Note that in smooth regions of the flow the ASLIP limiter is virtually the same as the SLIP limiter, but in non-smooth regions the ASLIP limiter becomes asymmetric about $i+1 / 2$ due to the independent action of the two halves, $L^{+}$and $L^{-}$, of operator $L$.

\section{Comparison of Baseline and SLIP/ASLIP Schemes}

A rigorous comparison of the above schemes is well beyond the scope of this paper, but a couple of preliminary comparisons are provided to demonstrate significant differences between the schemes. In particular, results for the nearly-incompressible laminar boundary-layer flow over a flat plate are presented for two conditions, freestream Mach numbers $M_{\infty}=0.30$ and $M_{\infty}=0.05$, respectively. For both flows the same grid was used and the Reynolds number based on plate length was $R e_{L}=1.0 \times 10^{6}$. Note that instead of RVC3D, a twodimensional CFD code [25] was used for these simulations, but the numerical formulations are very similar for both codes. Regarding the grid (not shown due to space limitations; 41 nodes normal to wall; 145 nodes along plate), it should be noted that grid characteristics such as coarseness, wall spacing, and wall stretching, were selected to be representative of typical RVC3D computations, and that these characteristics substantially influence the numerical solutions. Typical values for the numerical parameters were used as well $\left(\mu_{4}=1 / 32\right.$ for the baseline scheme, and $\mu_{4}=1$ for the SLIP and ASLIP schemes). Finally, it should be emphasized that the comparisons made here apply only to Mach numbers less than 0.5 .

Although the flow over the entire plate length was simulated in each case, it is sufficient to compare the results at only one plate location, $R e_{x}=5.0 \times 10^{5}$. Similar differences were observed at other plate locations.

The results for freestream Mach number $M_{\infty}=0.30$ are shown in Figure 1 where the normalized errors $\Delta u / u_{\infty}$ in the $u$-velocity profiles are compared for the baseline scheme (with and without preconditioning) and the SLIP and ASLIP schemes (only with preconditioning). A local error of zero corresponds to a local $u$-velocity which is equal to that of the "exact" Blasius solution. The dimensionless distance from the wall is the standard similarity parameter $\eta=(y / x) \sqrt{R e_{x} / 2}$.

The comparisons in Figure 1 reveal a relatively large difference in accuracy between the baseline and the SLIP/ ASLIP schemes, with the latter performing considerably better. It is noteworthy that the baseline scheme is improved substantially by the use of preconditioning, which in this case reduces the artificial dissipation (see Equations 25 and 26) due the to rescaling of eigenvalues (compare Equations 11 and 23). Although outside the scale of the graph, the maximum error of around -7.5 percent for the baseline scheme without preconditioning is 


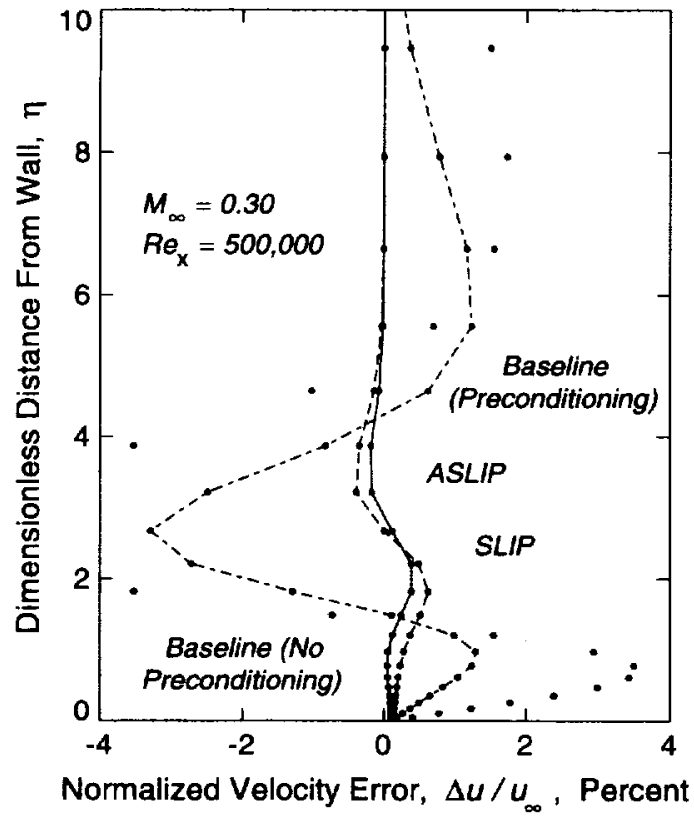

Figure 1 - Error In computed solutions of laminar Blasius velocity profiles using the baseline, SLIP, and ASLIP schemes $\left(M_{\infty}=0.30\right)$

reduced down to about -3 percent with preconditioning. This result illustrates the fact that at low Mach numbers the preconditioning methods being implemented favorably alter the steady-state solution, as noted earlier. The SLIP and ASLIP schemes both perform well, the ASLIP scheme being slightly more accurate than the SLIP. This small difference has been confirmed in general for a wide range of (undocumented) practical low Mach number simulations for various turbomachinery cases. It should be noted, however, that the ASLIP flux limiter has been observed to work well only for low Mach number flows, whereas the SLIP fiux limiter has a far wider range of application [20-22]. Finally, it can be seen in Figure 1 that the SLIP and ASLIP schemes completely eliminate the velocity "overshoot" oscillation at the outer edge of the layer, an effect commonly observed with respect to the baseline scheme at virtually all Mach number conditions. For a transonic airfoil example involving such an overshoot and its elimination by using the CUSP scheme with the SLIP flux limiter [20-22], see Reference 26.

Laminar boundary layer results similar to those above can be seen in Figure 2 for the freestream Mach number $M_{\infty}=0.05$. In this figure, however, no curve is included for the baseline scheme without preconditioning since the low freestream Mach number makes that computation impractical. Qualitatively the results are the same as for

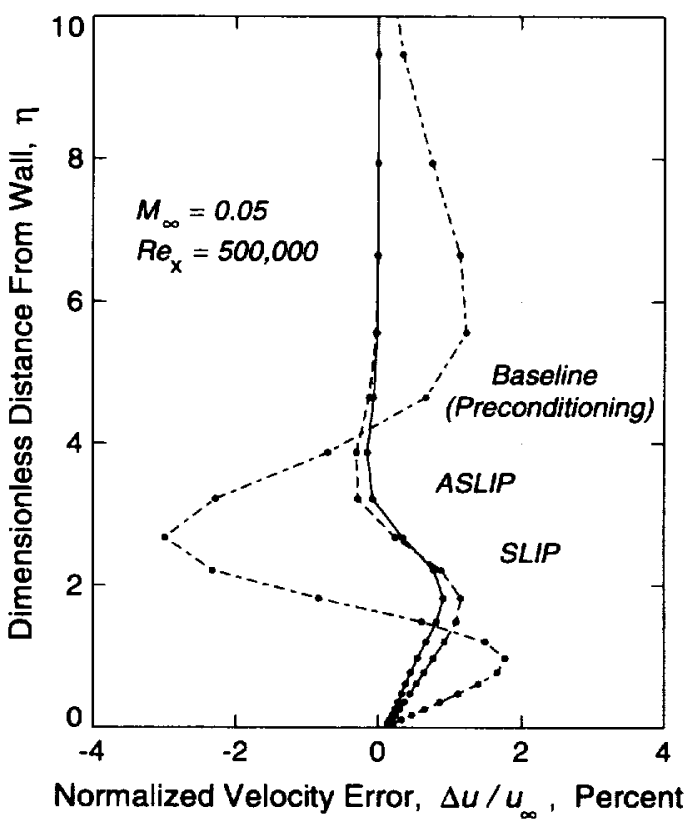

Figure 2 - Error In computed solutions of laminar Blasius velocity profiles using the baseline, SLIP, and ASLIP schemes $\left(M_{\infty}=0.05\right)$

$M_{\infty}=0.30$, although quantitatively the near-wall errors for the SLIP and ASLIP schemes are somewhat larger.

The above comparisons suggest that improved accuracy might be obtained in general by using the SLIP or ASLIP scheme rather than the baseline scheme. However, there are some disadvantages worth mentioning. A primary disadvantage is the direct increased computational overhead. For the 4-stage Runge-Kutta scheme with implicit residual smoothing, the overall CPU-time per iteration increases by about 26 percent for a typical low-speed viscous simulation, while for the 2 -stage Runge-Kutta scheme the increase is about 50 percent. Note that, as implemented in RVC3D, each scheme applies the artificial dissipation only once per time-step. Another notable disadvantage is that the SLIP and ASLIP schemes sometimes reduce the code stability with 4 -stage Runge-Kutta timestepping, although the most frequent adverse effect is just poor convergence behavior. The exact cause of this effect has not been determined, but it is thought that a multigrid scheme (not in RVC3D) might eliminate or reduce it while also improving code stability. Interestingly, good stability and convergence behavior is always achieved when using the SLIP and ASLIP schemes in connection with 2-stage Runge-Kutta time-stepping where, with implicit residual smoothing, CFL numbers of 2.5 to 3.0 can be routinely used. 


\section{Numerical Source Terms}

Application of the RVC3D code to low Mach number flows revealed that some approximations related to the finite-differencing were inadequate, even though no significant effects were encountered at higher Mach numbers where the code has been routinely applied. As a result, two basic modifications were necessary in order to avoid substantial numerical sources and get good convergence behavior with undistorted flow field solutions. First, the expressions for the finite-difference metrics had to be replaced with more rigorously formulated (and consequently more complicated) ones. Second, a corrective source term had to be added to account for errors introduced by coordinate-system rotation.

\section{Finite-Difference Metrics}

Until recently the RVC3D code used second-order central differences to evaluate the metric terms in Equation 7. However, as was pointed out years ago by Pulliam et al. [27], in three dimensions this scheme can introduce a freestream error, although it is noteworthy that Reference 27 mentions no discernible effects from ignoring the numerical source error other than the inability to maintain the free stream. At low enough Mach numbers, however, the resulting numerical sources are large enough compared to the physical flux terms to produce excessive flow field distortions, as well as hinder or prevent numerical convergence.

Metric finite-difference formulas based on cell-face projected areas have been in existence for some time, and can be found clearly presented in Reference 28 . For convenience, the numerical formulas are also given here:

$$
\begin{aligned}
& 2 J^{-1} \xi_{x}=\Delta_{y(0,1,1)} \Delta_{z(0,-1,1)}-\Delta_{y(0,-1,1)} \Delta_{z(0,1,1)} \\
& 2 J^{-1} \xi_{y}=\Delta_{z(0,1,1)} \Delta_{x(0,-1,1)}-\Delta_{z(0,-1,1)} \Delta_{x(0,1,1)} \\
& 2 J^{-1} \xi_{z}=\Delta_{x(0,1,1)} \Delta_{y(0,-1,1)}-\Delta_{x(0,-1,1)} \Delta_{y(0,1,1)} \\
& 2 J^{-1} \eta_{x}=\Delta_{y(1,0,1)} \Delta_{z(1,0,-1)}-\Delta_{y(1,0,-1)} \Delta_{z(1,0,1)} \\
& 2 J^{-1} \eta_{y}=\Delta_{z(1,0,1)} \Delta_{x(1,0,-1)}-\Delta_{z(1,0,-1)} \Delta_{x(1,0,1)} \\
& 2 J^{-1} \eta_{z}=\Delta_{x(1,0,1)} \Delta_{y(1,0,-1)}-\Delta_{x(1,0,-1)} \Delta_{y(1,0,1)} \\
& 2 J^{-1} \zeta_{x}=\Delta_{y(1,1,0)} \Delta_{z(-1,1,0)}-\Delta_{y(-1,1,0)} \Delta_{z(1,1,0)} \\
& 2 J^{-1} \zeta_{y}=\Delta_{z(1,1,0)} \Delta_{x(-1,1,0)}-\Delta_{z(-1,1,0)} \Delta_{x(1,1,0)} \\
& 2 J^{-1} \zeta_{z}=\Delta_{x(1,1,0)} \Delta_{y(-1,1,0)}-\Delta_{x(-1,1,0)} \Delta_{y(1,1,0)}
\end{aligned}
$$

where the difference operator is defined as

$$
\Delta_{x(I, J, K)}=\frac{1}{2}\left(x_{i+I, j+J, k+K}-x_{i-I, j-J, k-K}\right)
$$

Note that the indices $i, j$, and $k$ correspond to the coordinate directions $\xi, \eta$, and $\zeta$, respectively, and that these expressions for the metrics are fully conservative.

The metric Jacobian $J$ is calculated as before, using central-finite-differences, since it does not significantly influence the steady-state solution. However, unlike the case of a stationary coordinate system there is some (negligible) influence due to the rotation source term on the right-hand-side of Equation 1.

\section{Coordinate-System Rotation}

The numerical source error due to coordinate-system rotation can be examined analytically by considering a special case; namely, that of steady uniform flow in the $x$ direction, in which case the velocity component $u$ is a constant, the components $v$ and $w$ are zero, and all flow derivatives are zero. The continuity, $x$-momentum, and energy equations (see Equation 1) can then be reduced to the following expression:

$$
\begin{array}{r}
0=\Omega\left[\partial_{\xi}\left(-J^{-1} \xi_{y} z+J^{-1} \xi_{z} y\right)+\right. \\
\partial_{\eta}\left(-J^{-1} \eta_{y} z+J^{-1} \eta_{z} y\right)+ \\
\left.\partial_{\zeta}\left(-J^{-1} \zeta_{y} z+J^{-1} \zeta_{z} y\right)\right]
\end{array}
$$

The right-hand side of Equation 43 is mathematically equivalent to

$$
\frac{\Omega}{J}\left(-\frac{\partial z}{\partial y}+\frac{\partial y}{\partial z}\right)
$$

which is identically zero for any non-zero angular velocity $\Omega$. For the numerical formulation the right-hand side is not exactly zero, however, which produces a freestream error.

Using the result of Equation 43, and denoting the right-hand side as $S_{\Omega}$, a simple and straightforward source term correction can be applied in Equation 1 . In particular, an additional source term $\hat{K}$ can be included to exactly cancel the freestream error:

$$
\begin{array}{r}
\partial_{1} \hat{q}+\partial_{\xi} \hat{E}+\partial_{\eta} \hat{F}+\partial_{\zeta} \hat{G}-\operatorname{Re}^{-1}\left(\partial_{\eta} \hat{F_{V}}+\partial_{\zeta} \hat{G_{V}}\right) \\
=\hat{H}+\hat{K}
\end{array}
$$

where

$$
\hat{K}=S_{\Omega} q
$$


Note that for efficiency, $\hat{K}$ is only evaluated at the first stage of the Runge-Kutta scheme and then held constant along with the artificial dissipation and the viscous residual.

\section{Numerical Results}

The preconditioned RVC3D code has been applied to a well-documented experimental test case involving a large low-speed centrifugal compressor (LSCC) impeller tested at the NASA Lewis Research Center. After describing the experimental test facility along with the CFD grid, several computational details are discussed, followed by comparisons of computational and experimental results.

\section{Low-Speed Centrifugal Compressor Impeller}

The LSCC is a large-scale low-speed compressor test rig designed to duplicate the essential flow field and flow physics of a high-speed centrifugal compressor impeller [29-31]. Due to its large size and low speed, extensive aerodynamic instrumentation of the impeller was made feasible, as were detailed flow field measurements using a laser anemometer system [31].

In order to provide an illustration of the test impeller, a coarse rendering of the CFD grid on the impeller hub and blade surfaces is shown in Figure 3. The impeller has 20 full blades with 55 degrees backsweep and a design tip speed of $153 \mathrm{~m} / \mathrm{s}(1917 \mathrm{mpm})$. The inlet diameter is $0.870 \mathrm{~m}$ with an inlet blade height of $0.218 \mathrm{~m}$, and the exit diameter is $1.524 \mathrm{~m}$ with an exit blade height of $0.141 \mathrm{~m}$. The tip clearance between shroud and impeller is uniform at $2.54 \mathrm{~mm}$, which is 1.8 percent of blade height at the impeller exit. Fillet radii at the impeller hub are uniform

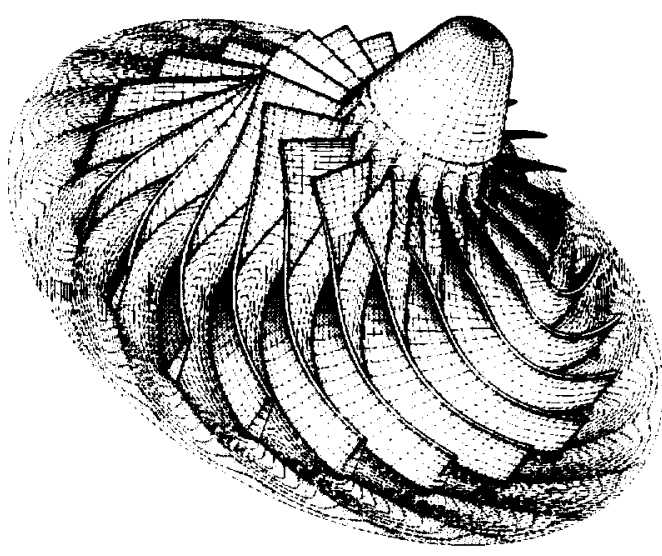

Figure 3LSCC impeller with coarse rendering of computational grid on hub and blade surfaces at $9.525 \mathrm{~mm}$. A vaneless radial diffuser is located downstream of the impeller.

Although Figure 3 shows all 20 impeller blades, the computational grid for RVC3D has only one blade (and blade channel), with blade-to-blade flow periodicity enforced by the boundary conditions. The RVC3D code uses C-type grids [11,12], and the same grid was used for all computational results presented below. The blade-toblade grid near midspan is shown in Figure 4 which includes expanded views for the blade leading- and trailing-edges. The dimension of the grid in the $\xi$-direction (wrapping around the blade) is 225 , while in the $\eta$-direction (normal to the blade surface) the grid has a dimension of 33 , giving a total of 65 nodes blade-to-blade. In the $\zeta$ direction (spanwise, hub-to-shroud), the grid dimension is 65 with more clustering near the shroud for better resolution of the blade tip clearance flow. As shown in Figure 5, the grid was stretched into the clearance gap region over the blade tip so as to provide for a direct simulation of the tip clearance flow, but without having to use a multi-block flow solver. The blade-hub fillets were not included in the

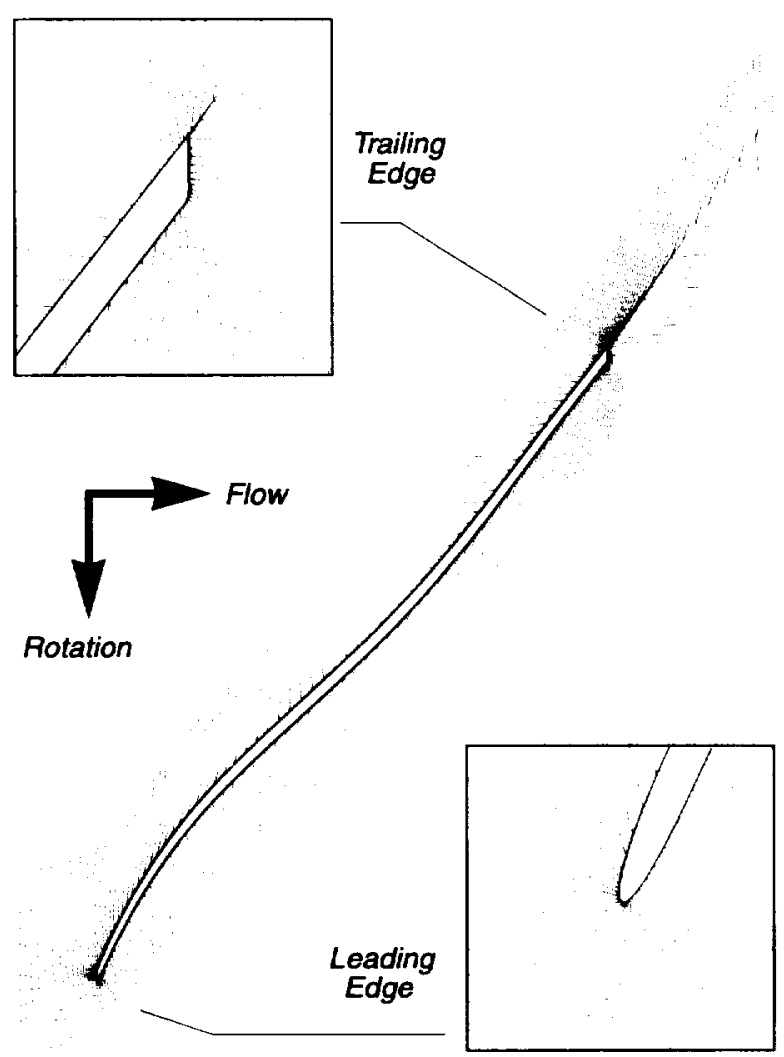

Figure 4 - Near midspan blade-to-blade view of computational grid for LSCC impeller 




Figure 5- Computational grid stretching into shroud clearance gap over blade tip

computational grid geometry since they are thought to have only a small influence on the impeller performance and flow field.

\section{Near-Design Operating Condition}

The compressor operating condition of interest for the present study is a near-design point for which extensive measurement data were obtained. The corrected flow rate at the near-design point was $30.0 \mathrm{~kg} / \mathrm{s}$ with the impeller rotating at $1862 \mathrm{rpm}$. Based on aerodynamic probe measurements, the overall impeller performance at that point has been reported [31] to be a total-pressure ratio of 1.141 with an adiabatic efficiency of 0.922 .

\section{Inlet Duct Flow Simulation}

To more accurately simulate the flow entering the impeller, while also eliminating several approximations often made at a rotor grid inlet boundary, an axisymmetric flow simulation for the entire inlet duct upstream of the impeller was performed by "loosely" coupling the duct fluid dynamic simulation to the impeller simulation. The coupling was implemented through the appropriate boundary conditions, and at the level of the computer operating system. Specifically, for every 150 iterations of the impeller simulation, a converged axisymmetric duct simulation was performed using the circumferentially-averaged spanwise static pressure distribution from the impeller grid inlet. The viscous two-dimensional CFD code used for simulating the axisymmetric duct flow is described in Reference 25. The resulting spanwise distributions of total pressure, total temperature, circumferential velocity, and meridional flow angle at the duct exit were then passed to the impeller grid inlet for the next block of 150 iterations. Good overall convergence between the duct and impeller solutions were obtained by under-relaxing the duct exit static pressure update at the beginning of each duct simulation.

Resulting velocity contours for the duct and impeller solutions as computed using preconditioning and the ASLIP artificial dissipation scheme (both solutions) are shown in Figure 6, where the velocities in the LSCC impeller are circumferentially-averaged and shown in the absolute frame-of-reference. Although mass-flow continuity was not strictly enforced between the duct and the impeller, the integrated values are nearly the same at 29.96 and $29.94 \mathrm{~kg} / \mathrm{s}$, respectively, which are within 0.2 percent of the measured flow rate.

\section{General Discussion of Impeller Simulations}

Several numerical simulations were performed for the LSCC impeller. It will be helpful to delineate them before proceeding to detailed comparisons of computational and experimental results. Numerical convergence issues will also be addressed in this context.

Two separate but related numerical scheme implementations are being examined in this study: namely, preconditioning and artificial dissipation. Use or non-use of the preconditioning with any of the artificial dissipation schemes discussed earlier leads to many possible combinations which could be compared. Due to scope and space limitations, however, only three are considered in this study:

- The baseline artificial dissipation scheme with no preconditioning. This case is possible due to sufficient compressibility of the flow being simulated, and allows comparisons of results computed with and without the preconditioner.

- The baseline artificial dissipation scheme with preconditioning.

- The ASLIP artificial dissipation scheme with preconditioning. The ASLIP was selected over the SLIP scheme since it is thought to be the most accurate at the low Mach number levels under consideration. However, the SLIP scheme generally produces results similar to those of the ASLIP scheme.

For the baseline case with preconditioning, as for the ASLIP case, the solution was obtained with coupling to the inlet duct flow. However, the duct solution is virtually the same as that shown in Figure 6 . The baseline case 


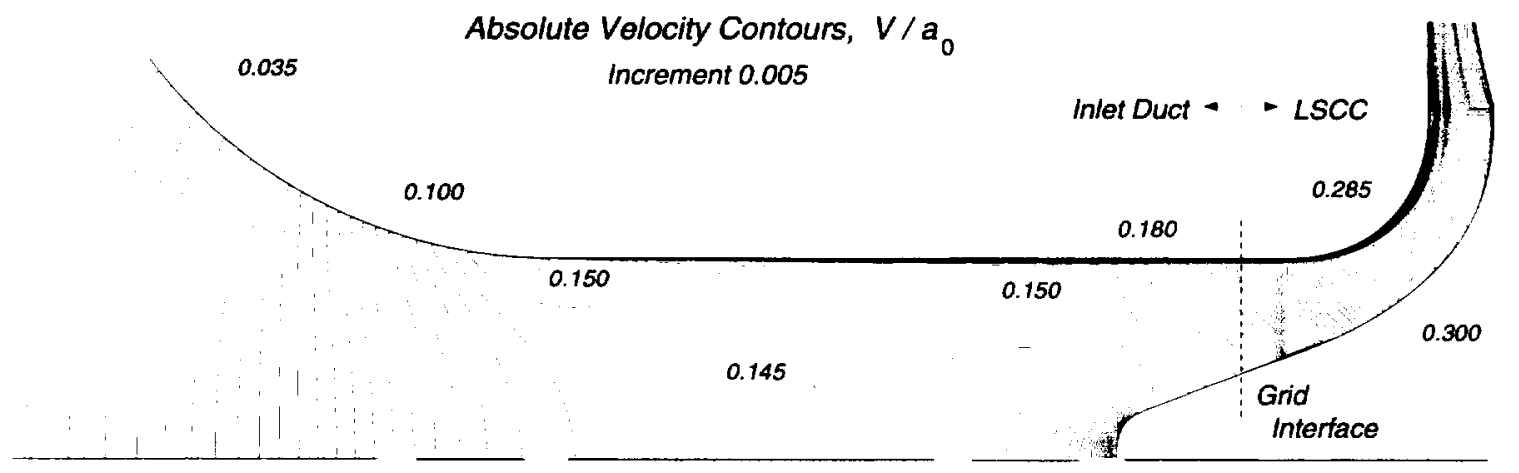

Figure 6 - Inlet duct and circumferentially-averaged LSCC impelier flow fields

with no preconditioning was solved without inlet coupling, using instead the inlet duct solution from the preconditioned baseline case.

In general, preconditioning can be expected to accelerate solution convergence for low Mach number flows, and if the Mach levels are low enough should allow a converged solution where none is possible without it. For the present case a convergence-history comparison is possible since the simulation converges without preconditioning. For the baseline artificial dissipation scheme $\left(\mu_{4}=1 / 32\right.$ and $\mu_{2}=1 / 4$ ), the benefit provided by preconditioning is shown in Figures 7 and 8. The normalized RMS-residual history in Figure 7 reveals a large acceleration in convergence over the first 2000 iterations. The accompanying variations in global mass-flow error and overall total-pressure ratio in Figure 8 are perhaps more revealing in that they show a dramatic acceleration from the preconditioning, such that by 1000 iterations the large oscillations in global mass-flow error and overall total-pressure ratio have been greatly diminished. It should be noted that both of these simulations were performed without coupling to the inlet duct in order to maintain a simple, direct comparison. They were executed using the 4-stage Runga-Kutta time-integration with implicit residual smoothing and a CFL number of 5.6. The computational overhead incurred by using the preconditioner for this simulation was determined on the CRAY C-90 to be about 22 percent, but as can be seen in the figures the convergence rate has been more than doubled as a result. Actual execution speeds for the non-preconditioned and the preconditioned solutions on the CRAY C-90 (using a single CPU) were 2.17 and $2.64 \mathrm{sec} /$ iteration, respectively.

As noted earlier, the use of the ASLIP (or SLIP) artificial dissipation scheme with the 4-stage Runga-Kutta time-integration often results in poor convergence behavior. Although the effect seems more associated with

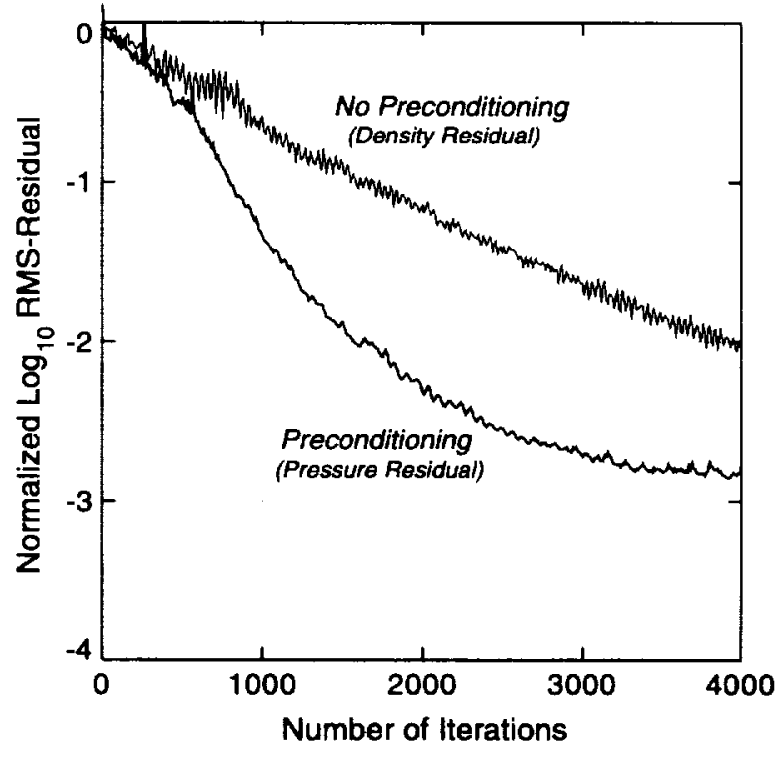

Figure 7 - Comparison of residual histories for aimulations with and without preconditioning (baseIine artificial dissipation scheme)

the residual history rather than the overall flow solution that is, a relatively small local convergence problem causes the residuals to "hang up" - the problem is completely avoided by using a 2-stage Runga-Kutta scheme where, with implicit residual smoothing, a CFL number of 3.0 can typically be used. In general, the use of the 2stage Runge-Kutta solver requires roughly 50 percent more CPU time (by requiring more iterations) to achieve a converged solution. For the LSCC impeller on the CRAY C-90, the execution speed with the ASLIP scheme and preconditioning was $2.67 \mathrm{sec} /$ iteration for the 2-stage 

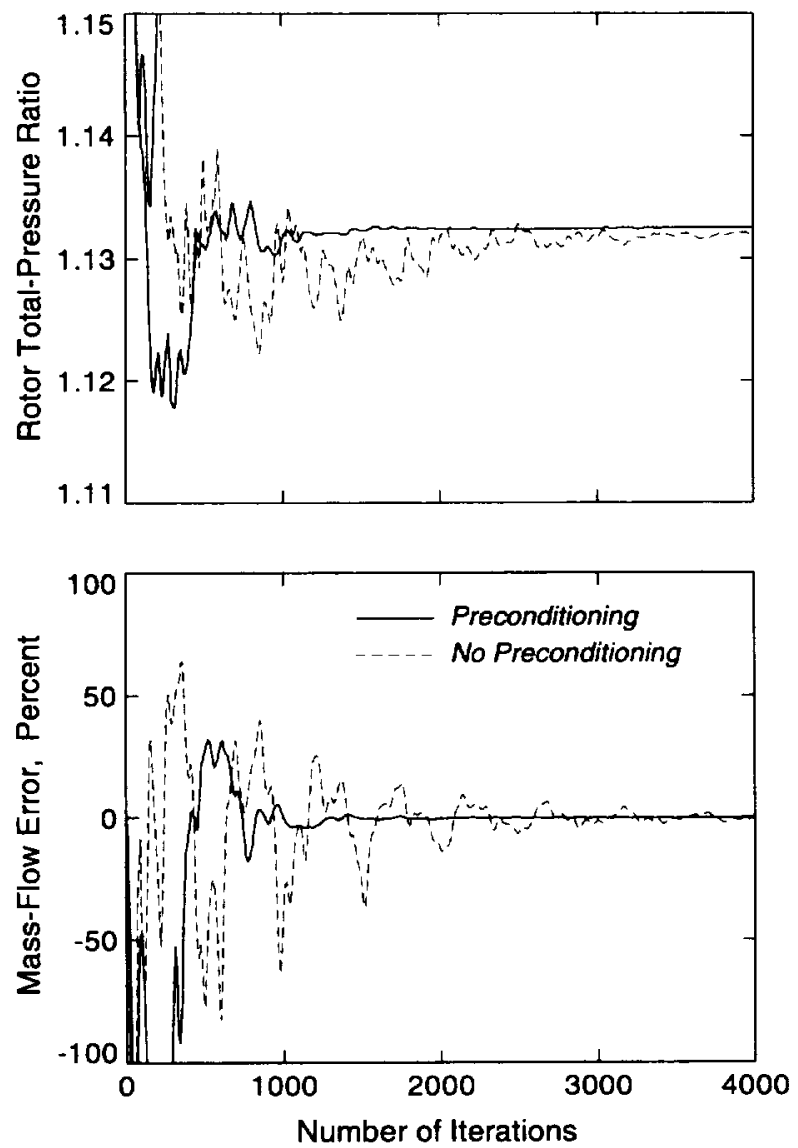

Figure B - Comparison of convergence histories for simulations with and without preconditioning (baseline artificial dissipation scheme)

solver, and about $3.31 \mathrm{sec} /$ iteration with the 4-stage solver. Note that all of the ASLIP results presented below were obtained using the 2-stage solver, whereas the baseline cases were run using the 4-stage solver.

\section{Detailed Comparisons of Results}

The computational results are compared with experimental data and with each other at several locations within or near the LSCC impeller. The experimental measurement locations of interest are shown in Figure 9 where the measurement station $J$-numbers are indicated for later reference. Computational results are first compared with experimental data at the two measurement stations upstream of the impeller ( $J=23$ and 48 ) in order to establish that the computed and measured impeller inflow conditions were in fact nearly the same.
Spanwise distributions of meridional velocity $V_{m}$ nondimensionalized by impeller tip speed $U_{t i p}$, and meridional flow angle $\bar{\alpha}_{m}$ are compared at station 23 in Figure 10, and at station 48 in Figure 11. As Figure 9 reveals, the computed solution at station 23 is within the inlet-duct domain since the grid interface (between the duct grid and the impeller grid) is downstream of station 23. Conversely, station 48 is located within the impeller-grid domain and is just upstream of the impeller leading edge. Since all three computed solutions are similar upstream of the impeller, only one computed result (ASLIP scheme) is shown for each station. The experimental data were acquired with a laser Doppler velocimeter (LDV), and circumferentially-distributed measurements have been circumferentially mass-averaged to obtain the results graphed. Generally close agreement between the numerical and experimental results can be seen in the comparisons, confirming the accuracy of the computed impeller inflow.

As the flow approaches and enters the impeller it accelerates and therefore undergoes some drop in static pressure. Within the impeller, however, there is a steady rise in both static and total pressure as the flow moves

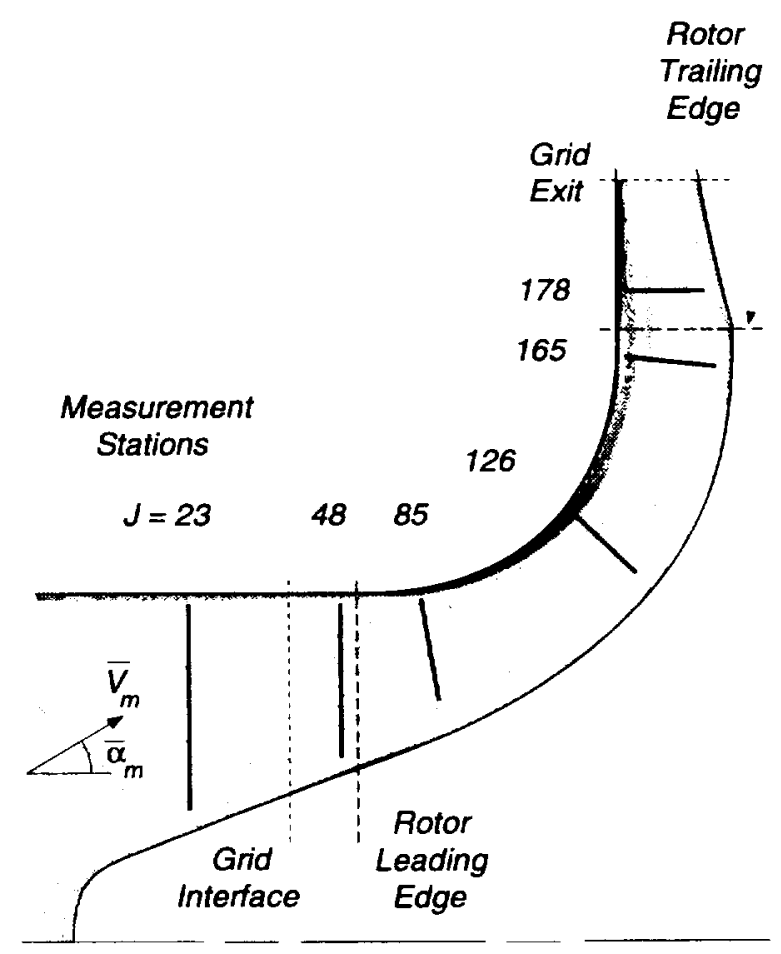

Figure 9- Experimental measurement station locations 


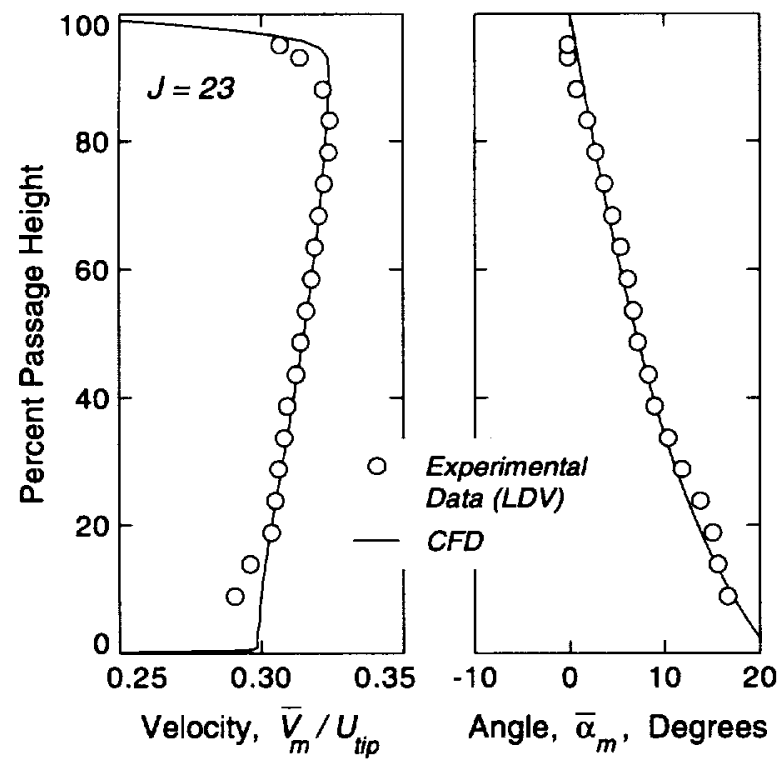

Figure 10 - Comparison of computed and measured spanwise profiles at impeller inlet station 23

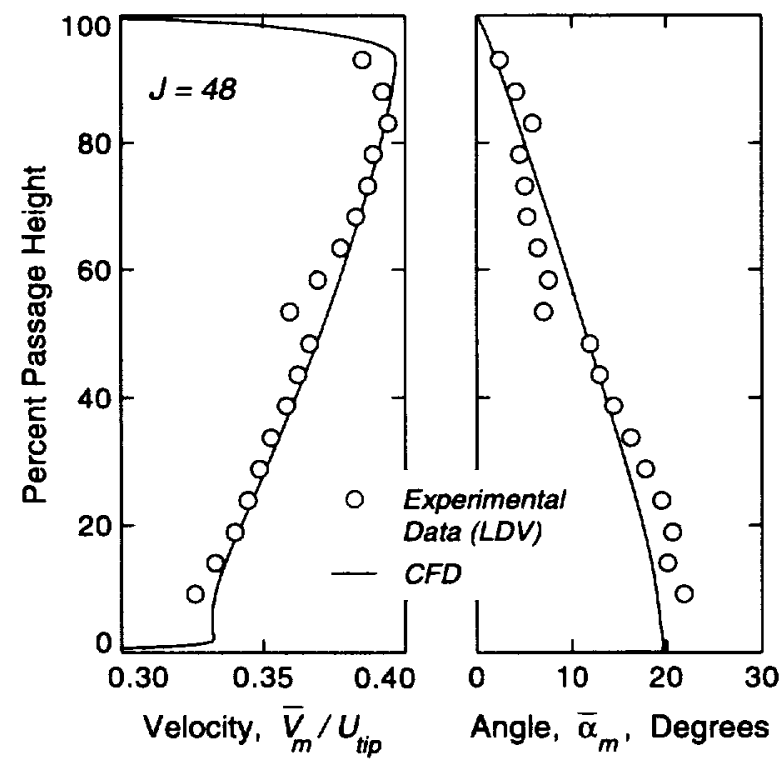

Figure 11 - Comparison of computed and measured spanwise profiles at Impeller Inlet station 48 through it and the impeller does work on the fluid. These features are readily apparent in Figure 12 which compares the measured steady-state shroud (tip) static pressure distribution through the impeller, to the computed and circumferentially-averaged shroud static pressure distribution. The pressures have been normalized by the inlet total pressure $p_{0}$, and the results are graphed as a function of meridional distance $m$ (from the impeller leading edge) normalized by the total meridional distance through the impeller blades $\Delta m_{\text {blade }}$. As with the inlet profiles, the differences between the various computed solutions is negligibly small so only one of them need be presented. It can be seen that the agreement between computation and experiment is very good, except perhaps at the impeller exit ( $m / \Delta m_{\text {blade }} \geq 100$ percent) where some differences are apparent.

Considerably more information about the pressure field within the impeller can be obtained from the static pressure distributions for the impeller blade surfaces. Blade surface pressure distributions for six different spanwise locations, namely $5,20,49,79,93$, and 97 percent span from the hub, are compared in Figure 13. Again, the differences between the three computed solutions are small enough to be ignored in this figure. As can be seen, the agreement between computation and experiment is very good, except on the pressure surface (PS) near the blade trailing edge ( $m / \Delta m_{\text {blade }}>90$ percent) where sig-

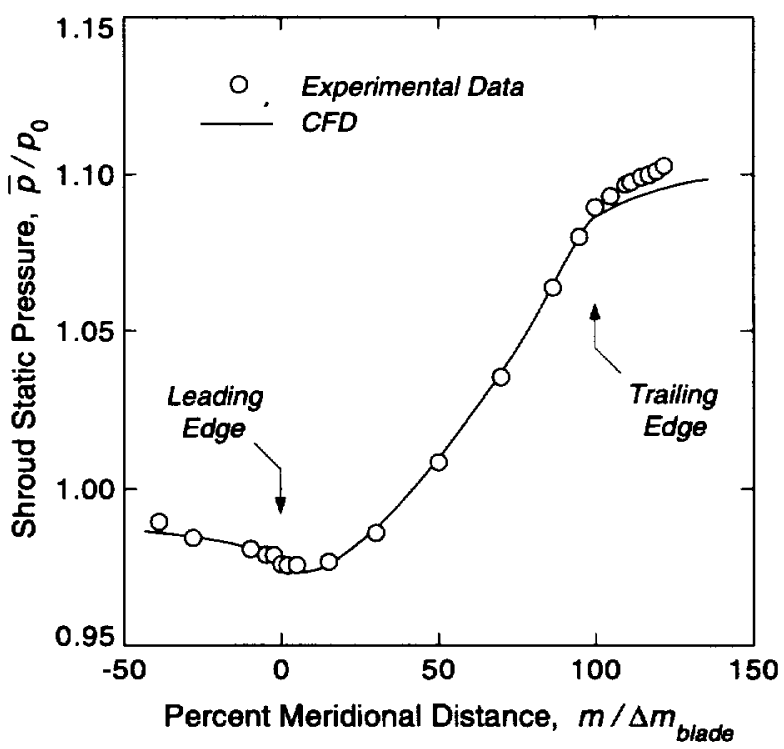

Figure 12 - Comparison of computed and measured shroud static pressure distributions 

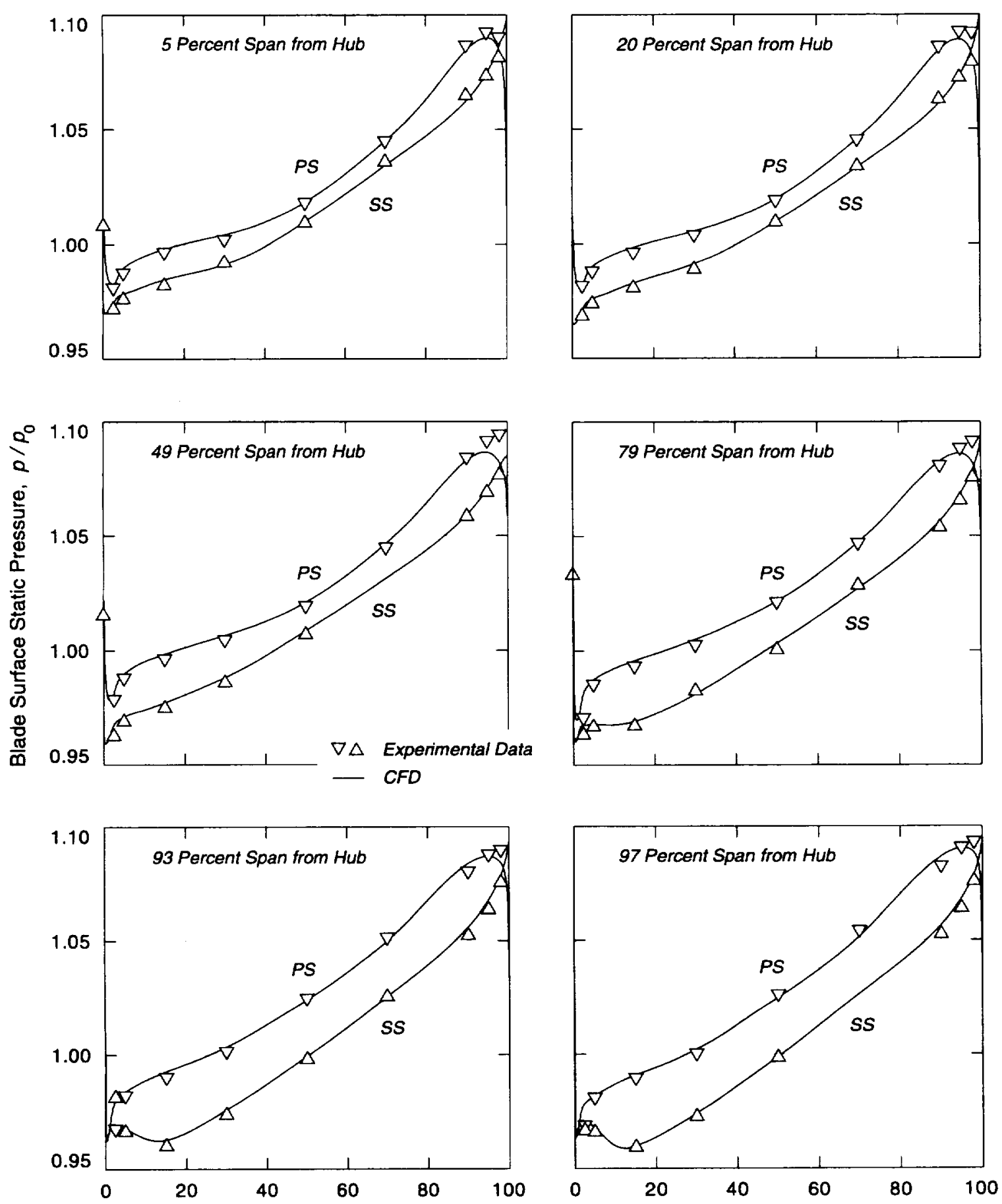

Percent Meridional Distance from Leading Edge, $m / \Delta m_{\text {blade }}$

Figure 13 - Comparison of computed and measured blade static pressure distributions at several spanwise locations 
nificant differences exist for most spanwise locations. Notice that at each spanwise location both the suction surface (SS) and pressure surface (PS) distributions are graphed together, which conveys useful information about the blade static pressure loading, the fundamental means by which the impeller does work on the fluid. The greater the area between the SS and PS curves, the greater the blade loading. With this in mind, examination of the graphs reveals that the experimental impeller has substantially more loading near the trailing edge than indicated by the numerical simulations. It can therefore be anticipated that the overall work input and total-pressure rise obtained from the computed results will be less than measured. Later it will be shown that this is actually the case.

In terms of the static pressure and inlet profile results presented so far, only results from one of the computed solutions (the ASLIP solution) were provided since, as noted, only minor differences existed between the three computed results. This does not imply, however, that only minor differences exist in general between the three solutions. In fact, significant differences do exist as will become apparent in much of what follows.

Contour plots of the blade-to-blade flow field at the 49 percent spanwise location from the hub are shown in Figure 14 for the three different computed cases (baseline with no preconditioning, baseline with preconditioning, ASLIP with preconditioning). Two sets of contour plots are compared: relative velocity $V^{\prime} / a_{0}$ on the left, and relative total pressure $p_{t}^{\prime} / p_{0}$ on the right. Note that the contour levels are not labeled in this figure since only a qualitative comparison is to be made. Close examination reveals that there are clear differences between the three solutions and, furthermore, that the differences are of such a character that a reasonable inference with regard to relative accuracy can be made.

Inspection of the relative total pressure contours in Figure 14 while taking into consideration certain basic fluid dynamic principles leads to a few useful conclusions. First, it can be pointed out that apart from any viscous flow effects the blade-to-blade contours should be straight, vertical lines in the figure. This follows from the fact that for a homentropic inlet flow without swirl the relative total enthalpy, and thus the relative total pressure, should depend only on radius; that is, the "rothalpy" is constant along an inviscid streamline. Viscous regions of the flow field such as the blade and endwall boundary layers, blade wakes, and any viscous secondary flows are expected to produce distortions in the contour shape. It can be seen in the figure that only the ASLIP solution nearly achieves the expected result - straight vertical lines between blade boundary layers - whereas the baseline scheme without preconditioning departs substantially from it. The corresponding differences in the midspan velocity field (Figure 14, left) are significant, especially in the exit region of the impeller. With preconditioning the baseline scheme is significantly better than without it, but noticeable errors in the total pressure contour shapes can still be seen. Also noticeable in the two baseline solutions but absent in the ASLIP solution is a total pressure oscillation (overshoot) at the outer edge of the blade boundary layers. The velocity contours also exhibit this overshoot, although it is less apparent. Recall that this behavior was shown and discussed earlier for the laminar flat plate boundary layer solutions (see Figures 1 and 2). In fact, the general trend in accuracy for the three LSCC impeller solutions parallels exactly that presented earlier for the flat plate.

Detailed comparisons of the computed and measured results downstream of the impeller at station 178 (see Figure 9) are presented next, followed by some comparisons at stations within the impeller. At this juncture it might be pointed out that in the aft half of the impeller strong viscous secondary flows develop and that these flows reside mostly in the outer one-third (shroud side) of the span while within the impeller. Downstream of the impeller, spanwise mixing and migration begin to redistribute the flow.

Spanwise distributions of steady-state total pressure $\bar{p}_{t} / p_{0}$ and static pressure $\bar{p} / p_{0}$ at station 178 are compared in Figure 15. Note that the hub and shroud correspond to the right and left sides, respectively, of each graph. The experimental data in this figure were obtained using slow-response pneumatic probes, and the computational results represent circumferentially mass-averaged solution data. Closely related to the total pressure distributions are the work-coefficient $\Delta\left(U \bar{V}_{\theta}\right) / U_{\text {tip }}^{2}$ distributions presented in Figure 16 where results for station 165 (see Figure 9) are included below those for station 178. Again, the computational results represent circumferential mass-averages, as do the LDV experimental data. The experimental "pneumatic probe" results in Figure 16 were calculated from measured total pressures using the wellknown Euler work equation, as described in Reference 31.

The static pressure distributions in Figure 15 (bottom) were determined primarily by the static pressure conditions imposed downstream, either by the throttle setting in the experiment or by the grid exit static pressure distribution in the computations. Slightly different grid exit static pressures, constant over the span, were specified for the three CFD cases so as to obtain the same mass flow rate. As can be seen in the graph, the experimental data indicate a slight positive pressure gradient from shroud-to-hub which is assumed real, but could be a measurement error due to probe immersion blockage effects - but the magnitude is small enough to be neglected. 


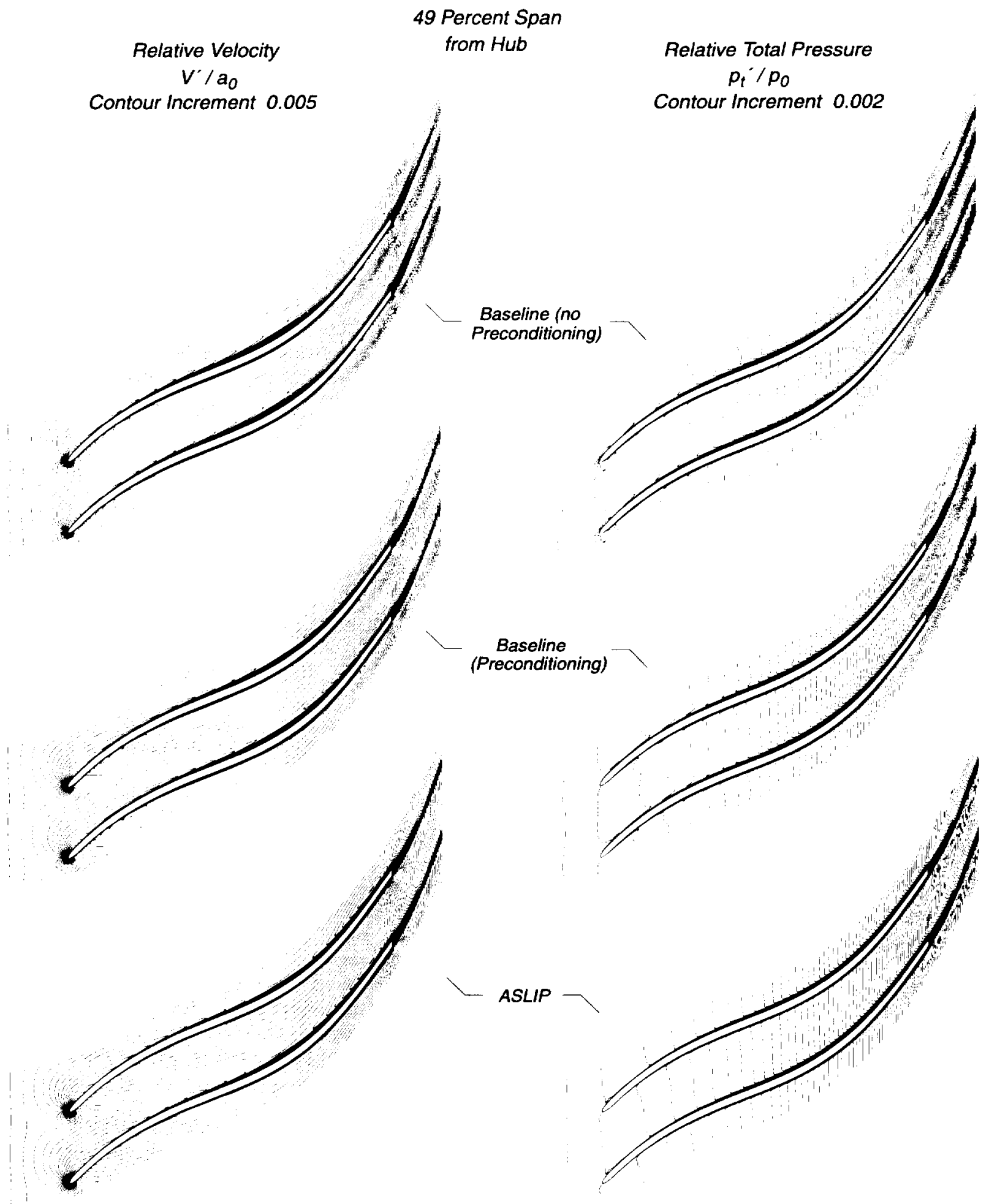

Figure 14 - Near-midspan contour plots of relative velocity (left) and relative total pressure (right) 

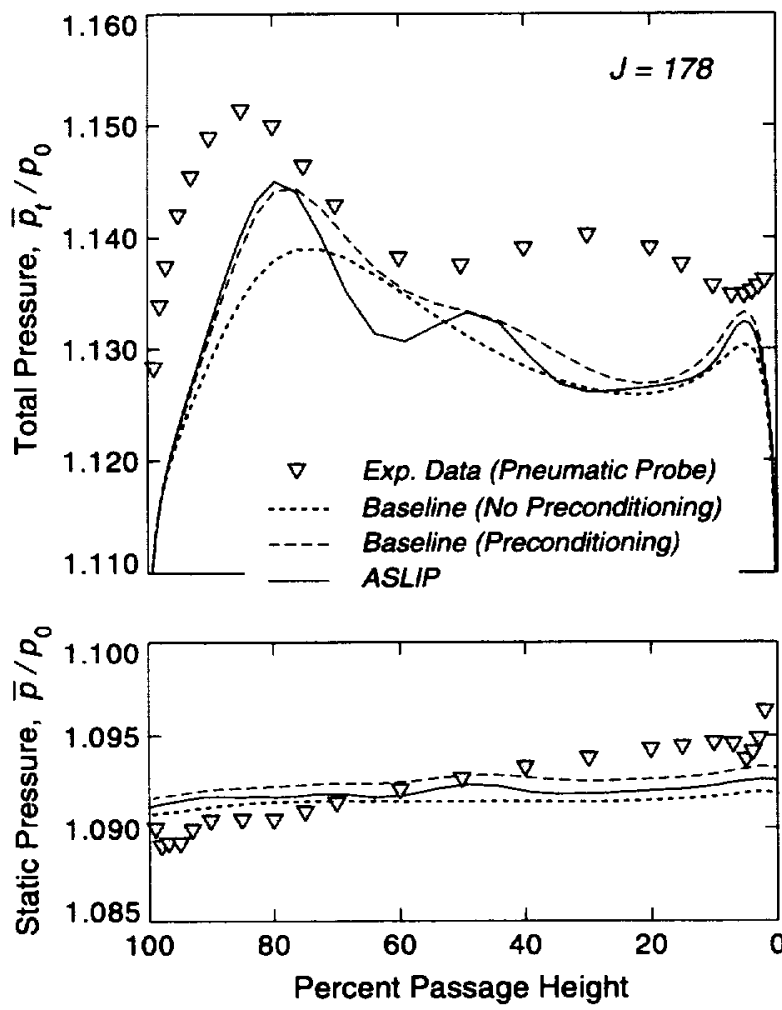

Figure 15 - Comparison of computed and measured spanwise total and static pressure distributions at station 178

The total pressure distributions in Figure 15 (top) reveal significant differences between the computed results, all three of which differ quite substantially from the experimental results. The work coefficient distributions in Figure 16 (top) show virtually the same trends and differences, which strongly implies that the differences in total pressure are due to differences in impeller work rather than in impeller total-pressure loss. As was pointed out earlier, the higher level of experimental impeller work is to be expected in view of the differences in blade (static pressure) loading (see Figure 13). Recall from Figure 13 that the experimental blade loading over the last 5 to 10 percent of blade chord (near the trailing edge) was discernibly higher than computed. In fact, closer examination of the pressure distributions in Figure 13 makes it clear that the simulated impeller undergoes a rapid aerodynamic unloading, even a negative loading, as the trailing edge is approached. This unloading is due mostly to an abrupt drop in static pressure on the blade pressure side. The experimental pressure surface data, however, do not show this.

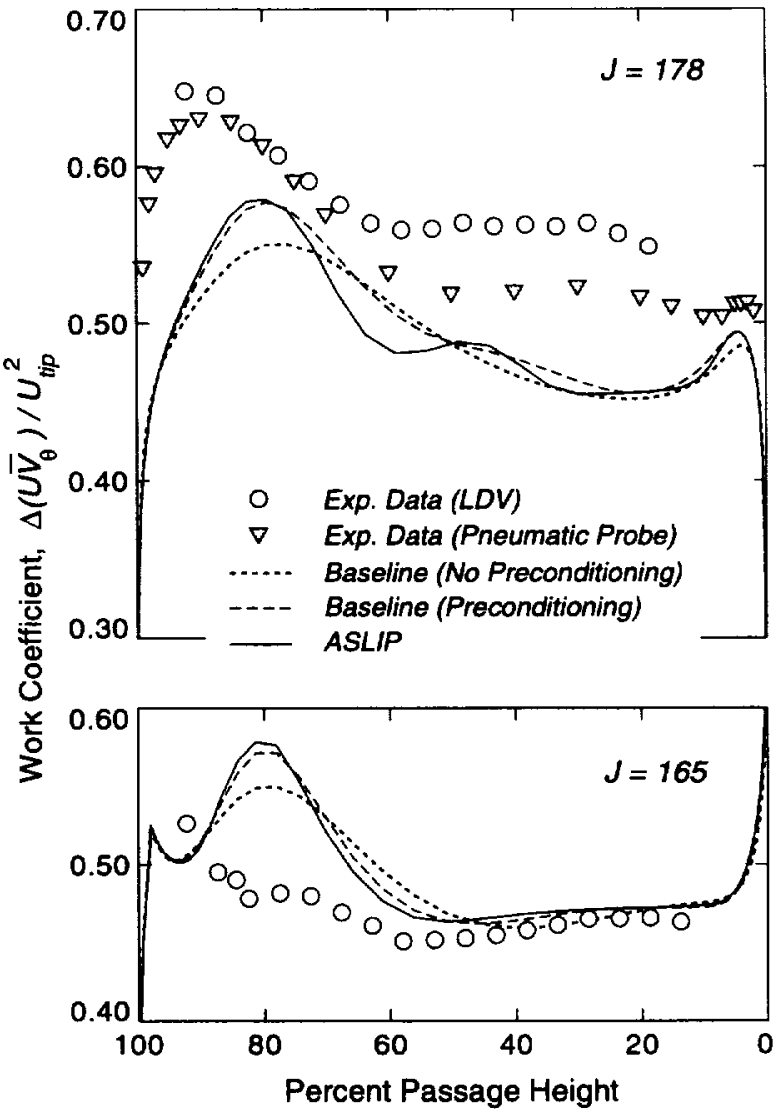

Figure 16 - Comparison of computed and measured spanwise work-coefficient distributions at stations 178 and 165

Further substantiation of the above discussion is provided by the spanwise work coefficient distributions at station 165 (see Figure 16, bottom), which is located in the impeller at about 94 percent of blade chord. At that location it can be seen that the agreement between computation and experiment is much better, except around 80 percent of passage height (span) where the influence of a viscous, secondary-flow tip-clearance vortex is strong.

More spanwise comparisons at stations 178 and 165 are given in Figure 17 ; radial velocity $\bar{V}_{r} / U_{t i p}$ on the top half, and relative flow angle $\bar{\beta}$ on the bottom half. Note again, that the quantities graphed are circumferential-averages, and that the flow angles were calculated using averaged circumferential and radial velocities:

$$
\bar{\beta}=\tan ^{-1}\left(\bar{V}_{\theta}^{\prime} / \bar{V}_{r}\right)
$$



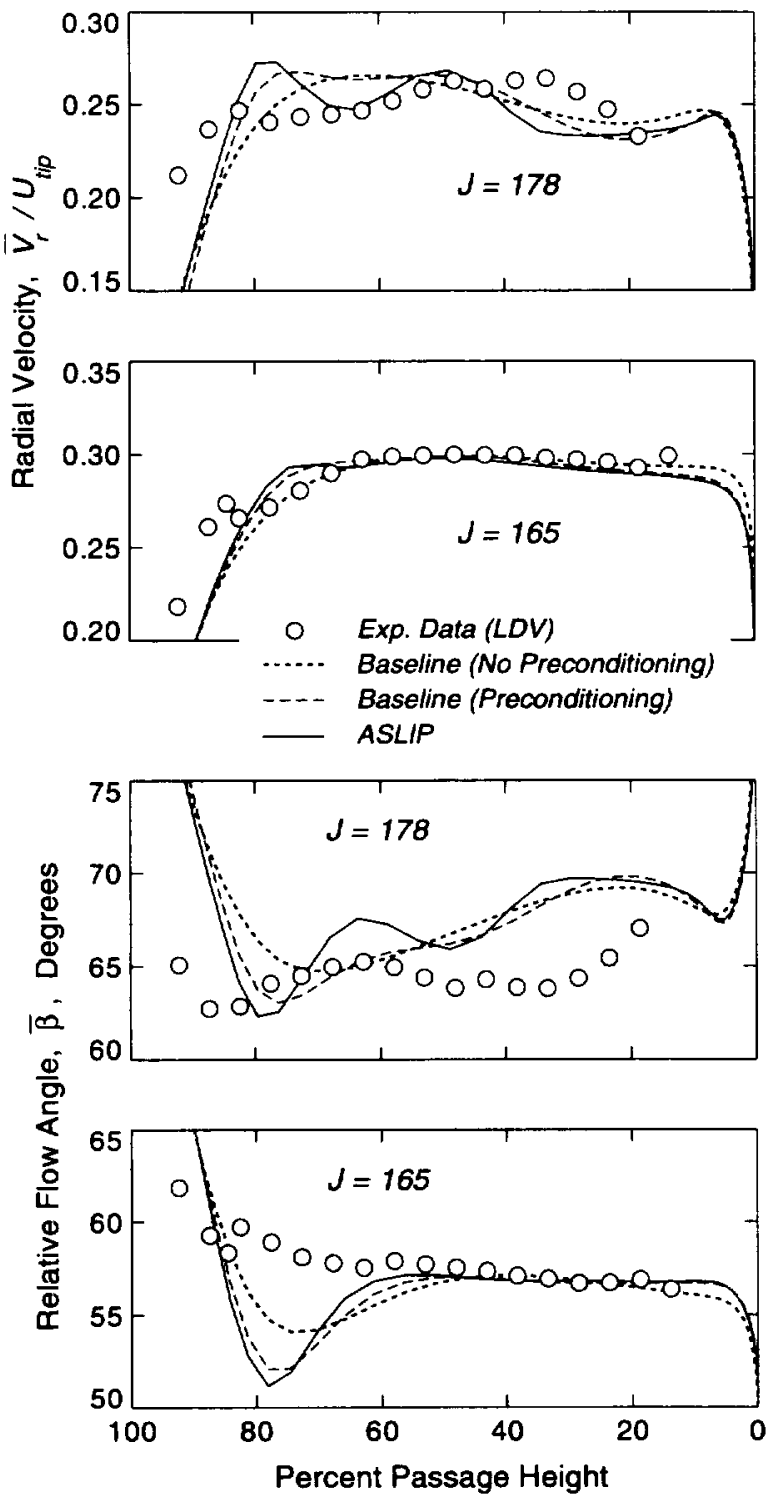

Figure 17 - Comparison of computed and measured downstream work-coetficient distributions

It might be added that at stations 165 and 178 , the radial velocity is approximately equal to the through flow velocity since the flow path is nearly radial.

As can be seen in Figure 17, the computed radial velocities at station 165 are in good agreement with each other and with the experimental data. At station 178 the agreement in radial velocity between computation and experiment is less favorable, but still good, and there are larger differences between the computed solutions. The relative flow angle distributions for station 165, like the radial and work coefficient (absolute tangential velocity) distributions, are also in reasonable agreement, except near 80 percent span (compare Figure 16, bottom). The differences between the computed and measured angle distributions at station 178 are merely a consequence of the differences in impeller work since the radial velocities are in fair agreement.

An attempt to understand and explain the underlying reason(s) for the discrepancies between computation and experiment at station 178 is well beyond the intent and scope of this study. Nevertheless, some further description and explanation should provide a better perspective to the comparisons being made, and might also help to stimulate more insight for future investigations.

An overall picture of what is happening at the impeller exit can be seen by considering a nominal velocity vector triangle for the exit flow. This consideration need only be qualitative, as in Figure 18 (top) showing the basic differences between the experimentally and computationally derived triangles. The triangles in the figure have been drawn such that the absolute tangential velocity $V_{\theta}$ (related to impeller work; see Figure 16, top) is larger for the experimental flow, and the radial velocity $V_{r}$ is about the same for computation and experiment (see Figure 17,
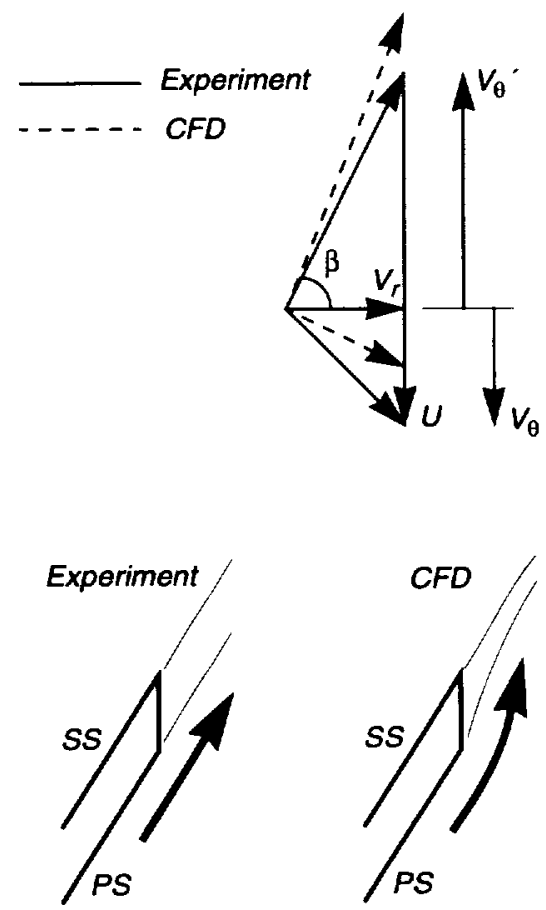

Figure 18 - Qualitative comparison of exit velocity vector trlangles and impeller trailing-edge flows 
top). As a result, the relative tangential flow velocity $V_{\theta}{ }^{\prime}$ and relative flow angle $\beta$ are smaller for the experimental flow (see Figure 17, middle bottom). Since the differences illustrated in Figure 18 are generated in just the aft 5 to 10 percent of the impeller, and since the computed pressuresurface pressures on the blade drop rather abruptly near the trailing-edge whereas the experimental pressures do not (see Figure 13), it can be inferred that the computed flow experiences a trailing-edge deflection away from the direction of impeller rotation ( $U$-direction) while the experimental flow does not, as illustrated in the lower half of Figure 18. The computed pressure drop indicates streamline curvature at the pressure-surface/trailing-edge corner accompanied by a local flow acceleration toward the trailing-edge. The absence of a drop in pressure for the measured pressure-surface data indicates that this acceleration with deflection does not occur in the experimental impeller.

The reason(s) why the numerical simulations fail to properly model the flow physics at the impeller exit is not presently known, but the authors feel confident that it is not due to the basic numerical scheme or approach; for example, grid density, Runge-Kutta time-integration, artificial dissipation scheme (all three artificial dissipation schemes fail almost equally), etc. An earlier, independent computational study by Hathaway et al. [32] on the same impeller revealed similar results using a different NavierStokes code with different computational grids. It is currently thought that some important physical effect has not been modeled adequately if at all, such as large-scale unsteadiness (vortex shedding), or that an important experimental boundary condition (related to the LSCC test rig) has possibly been neglected. The turbulence model may also be an important factor, especially as it relates to wake turbulence.

In any case, the relatively large differences between certain computational and experimental quantities at the impeller exit (station 178) make it more complicated to use the experimental data there to confirm or discredit the relative accuracy of the three CFD solutions. Fortunately, the results presented previously seem adequate to establish that the ASLIP scheme is by far the most accurate, followed by the baseline scheme with preconditioning. With this in mind it can be pointed out in Figures 15 through 17 that the more accurate the scheme, the finer the apparent resolution of viscous flow field features as revealed by spanwise undulations in the curves. These curves, however, represent circumferentially-averaged data, so it is of interest to examine and compare corresponding contour plots, as will be done next. These comparisons will further solidify the conclusions about scheme relative accuracy, as well as demonstrate that the impeller exit flow field computed using the ASLIP scheme is fairly accurate in many respects despite the problems delineated above.

Contour plots of radial velocity $V_{r} / U_{t i p}$ at the impeller exit (station 178, see Figure 9) are shown in Figure 19a where the dashed boxes enclose the area in which experimental LDV data were acquired. The experimental contours are shown at the top of the figure, followed underneath by the ASLIP, baseline with preconditioning, and baseline without preconditioning results. Very noticeable is the increase in flow-field resolution while progressing from the non-preconditioned, to the preconditioned baseline, to the ASLIP solution, the latter agreeing most closely with the LDV data. Qualitatively there are some features in the ASLIP solution not appearing in the experimental result, for example the low velocity zone near the upper-left corner of the dashed box, but overall the agreement is remarkably good. Also, where there is good qualitative agreement, the quantitative comparison is fairly close as well.

A similar figure is presented next, Figure $19 \mathrm{~b}$, which contains contour plots of relative circumferential velocity $V_{\theta}^{\prime} / U_{\text {tip }}$. The same general observations can also be made from this figure except, for reasons already discussed, those involving direct quantitative comparisons between computation and experiment. However, if a constant value of about 0.09 is subtracted from the CFD contours then the quantitative agreement is also fairly good for the ASLIP solution.

Finally, to complete the detailed comparisons several contour plots of the flow field at three locations within the impeller, stations 85, 126, and 165 (see Figure 9), will be presented. At these locations the experimental results will be compared only with the ASLIP results since they are the most accurate.

The first comparison at station 85 is near the inlet of the impeller, around 15 percent of meridional distance behind the impeller blade leading edges. At that location the flow is predominately axial and just a relatively small fraction of the total impeller work has been done on the fluid. Therefore only axial velocity $V_{x} / U_{\text {tip }}$ contours, shown in Figure 20, are compared there. In the figure, the ASLIP results are on the left while the experimental LDV results are within the dashed frame on the right. As can be seen, the agreement is very good qualitatively and quantitatively.

The next comparison at station 126 is just over halfway through the impeller, at about 56 percent of meridional distance from the blade leading edges. At this location contour plots for all three components of velocity, $V_{x} / U_{t i p}, V_{r} / U_{t i p}$, and $V_{\theta}^{\prime} / U_{t i p}$, are shown in Figure 21, again with the ASLIP results on the left and the LDV results in the dashed frame on the right. The agree- 


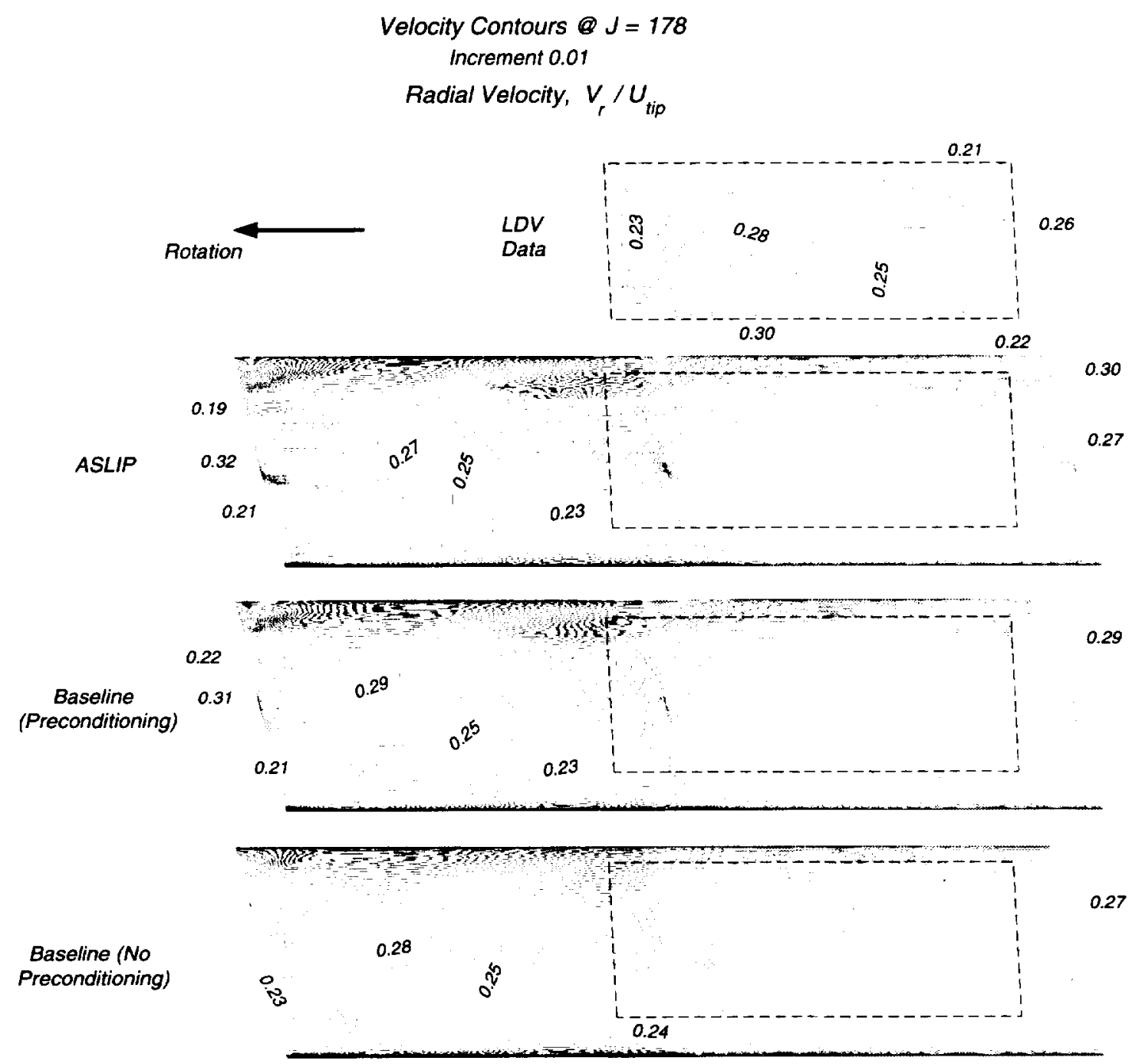

Figure $19 a$ - Measured and computed radial velocity contours at station 178

ment between computation and experiment is fairly good for all three components, although careful examination does reveal some minor differences.

The last comparison is near the exit of the impeller at station 165 where spanwise distributions of circumferentially-averaged velocities were presented earlier (see Figures 16 and 17). Contours of radial velocity $V_{r} / U_{t i p}$ and relative circumferential velocity $V_{\theta}^{\prime} / U_{t i p}$ are shown in Figure 22 in the same format used for the previous two figures. As can be seen, there is again close agreement between computation and experiment for both components, except within the tip-clearance vortex region located in the upper-right quadrant of the blade passage.
For radial velocity the discrepancies are relatively small, but for circumferential velocity a very substantial difference exists at the core of the vortex where viscous effects dominate. Specifically, in the computational result the relative circumferential velocity decreases monotonically toward the center on the region, reaching a minimum value of 0.21 . In the experimental result, however, the relative circumferential velocity first begins to decrease and then increases toward the center of the region which has a measured value of about 0.46 . This basic difference between computation and experiment was also apparent in Figures 16 (bottom) and 17 (bottom) at around the 80 percent span location. 


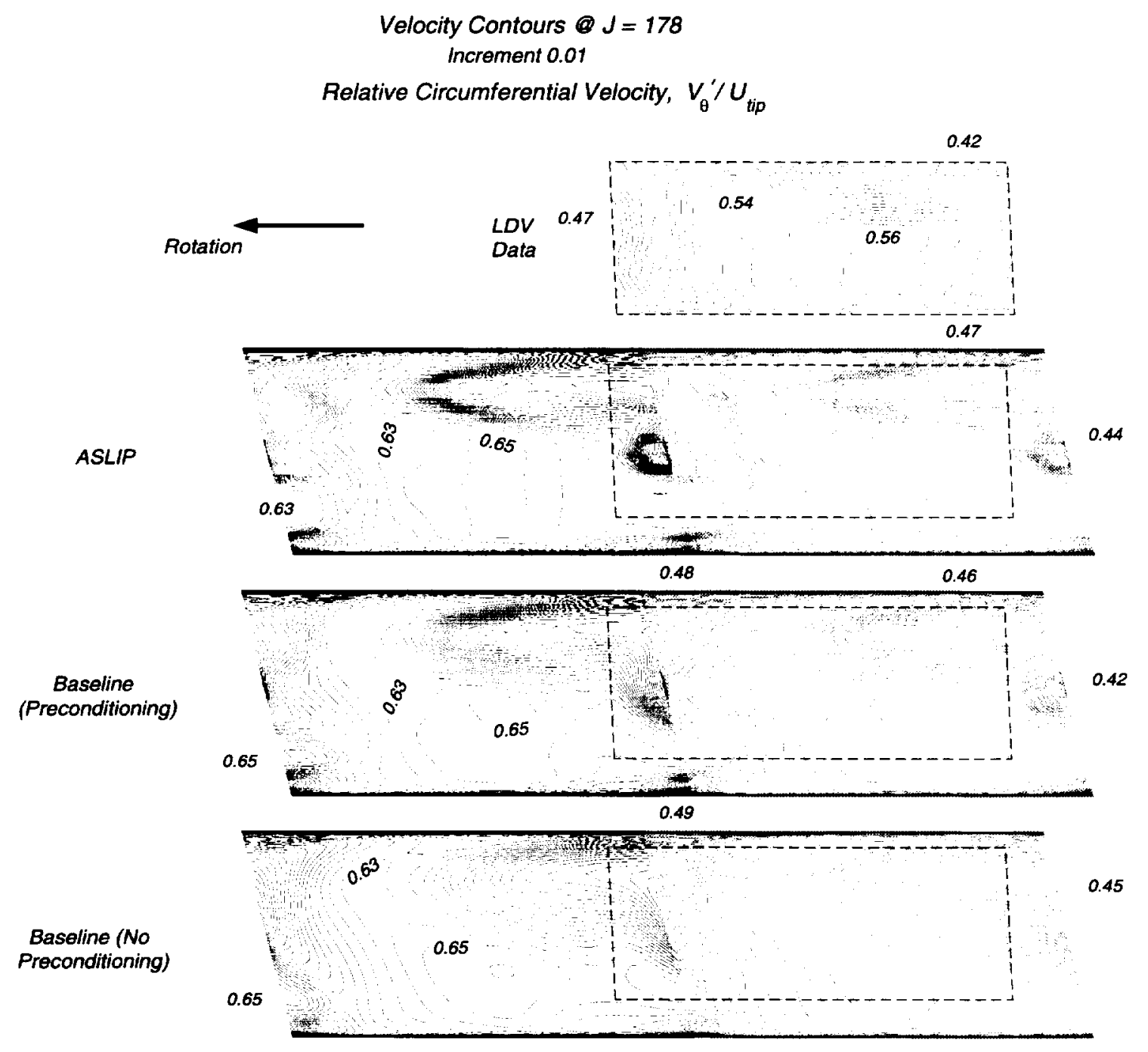

Figure 19b - Measured and computed relative clrcumferential velocity contours at station 178

\section{Conclusions}

A preconditioning scheme has been implemented into a three-dimensional viscous computational fluid dynamics code for turbomachine blade rows. The preconditioning allows the code, RVC3D, originally developed for simulating compressible flow fields, to be applied to nearlyincompressible, low Mach number flows. A brief description was given of the compressible Navier-Stokes equations for a rotating coordinate system, along with the preconditioning method employed. Details about the conservative formulation of artificial dissipation were provided, and different artificial dissipation schemes, namely the baseline scheme, the SLIP scheme, and the ASLIP scheme, were discussed and compared. A preliminary comparison for a laminar flat-plate boundary layer was made first, followed by the application of the preconditioned code to a well-documented case involving the NASA large low-speed centrifugal compressor for which detailed experimental data are available for comparison. The computational and experimental results, including performance and flow field data, were compared at the near-design operating point of the compressor.

Results for three numerical schemes were investigated in detail, the baseline (artificial dissipation) scheme without preconditioning, the baseline scheme with preconditioning, and the ASLIP scheme with preconditioning. Several conclusions can be summarized from the study: 
Velocity Contours@ $J=85$ increment 0.01

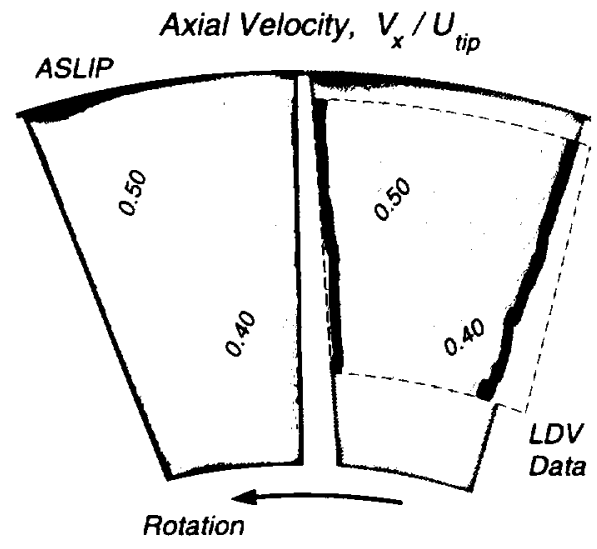

Figure $20-$ Measured and computed axial velocity contours at station 85

- Preconditioning greatly accelerates the convergence rate of the numerical simulation, while also improving the accuracy of the steady-state solution (for the low Mach number range investigated).

- The solution computed using the ASLIP scheme was considerably more accurate than the baseline solution (with preconditioning). The laminar boundary layer results revealed relative levels of accuracy and nonphysical flow features (velocity profile over-shoots) which were representative of the more-complicated viscous three-dimensional solution results. A disadvantage of the ASLIP scheme was poorer convergence behavior, resulting in longer solution times, but the improvement in accuracy is considered to be well worth the added computational overhead.

- In general, good agreement was observed between the computed (ASLIP scheme) and experimental results. An area of relatively poor agreement, the impeller blade loading and work at the impeller exit, was identified and addressed in some detail. It was suggested that the problem is possibly due to inadequate modeling of some important physical effect, or to the neglect of an important boundary condition present in the experimental test rig. Deficiencies in the turbulence model were also cited as a possible cause of the discrepancy.

In closing, it could be added that the LSCC impeller proved to be a good test case for the numerical investigation reported herein. The medium-low Mach number lev-
Velocity Contours @ $J=126$ Increment 0.01
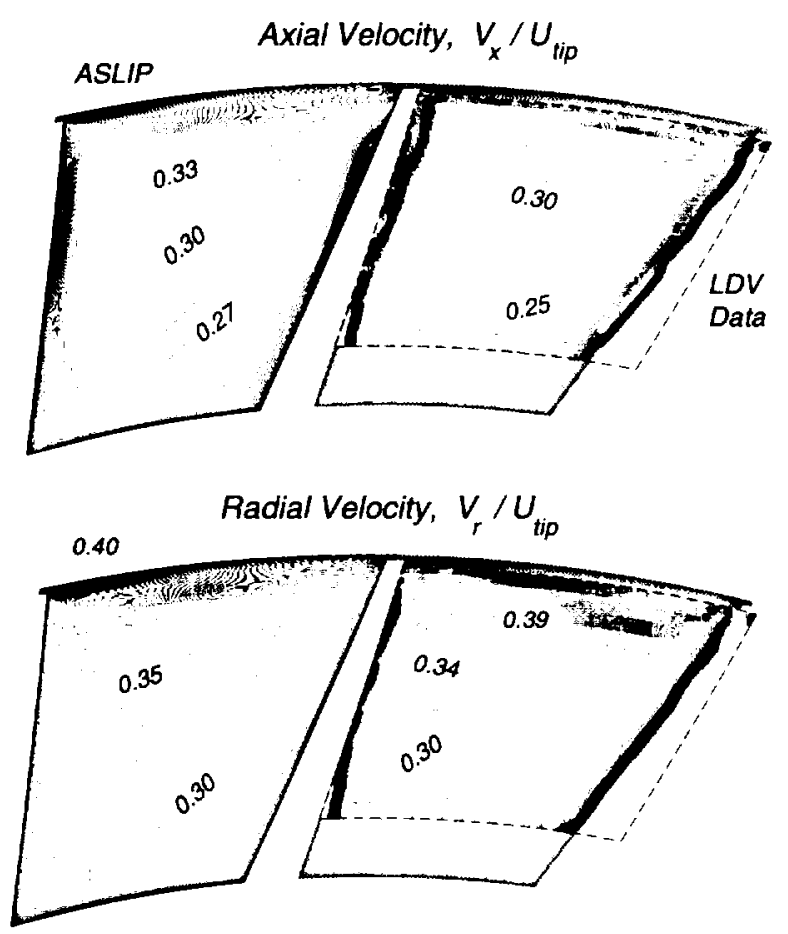

Relative Circumferential Velocity, $V_{\theta}^{\prime} / U_{\text {tip }}$

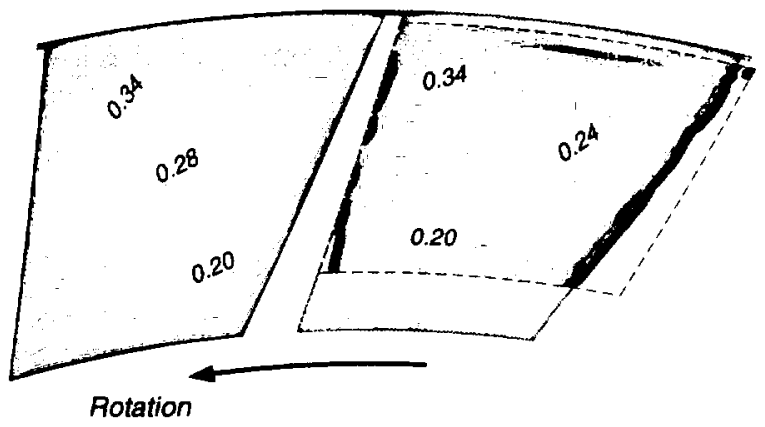

Figure 21 - Measured and computed axial, radial, and relative circumferential velocity contours at station 126 


\section{Velocity Contours@ $J=165$}

Increment 0.01

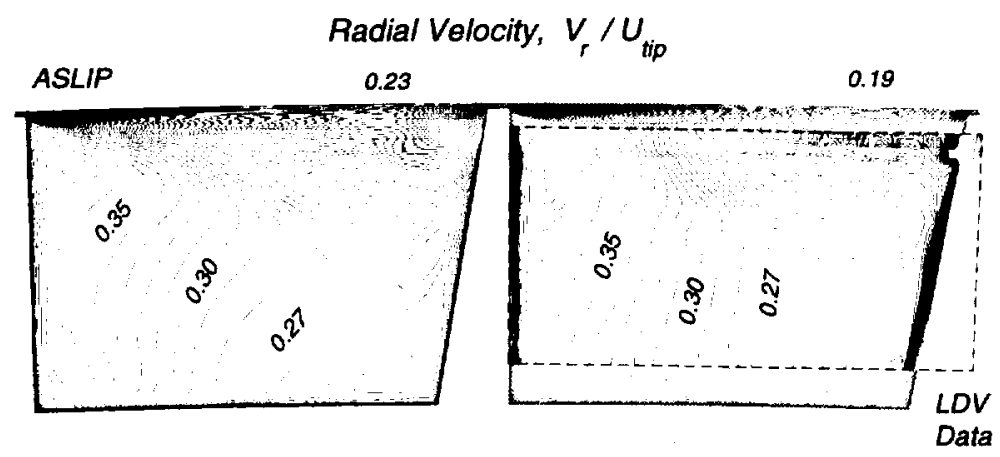

Relative Circumferential Velocity, $V_{\theta}^{\prime} / U_{t i p}$

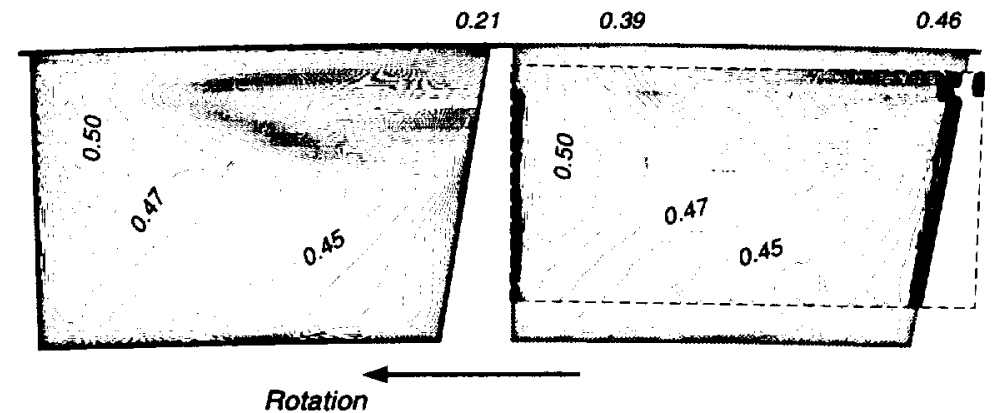

Figure 22 - Measured and computed radial and relative circumferential velocity contours at station 165

els made a comparison with the non-preconditioned solution practical, and the viscous-dominated flow field in the aft-half of the impeller exposed substantial differences which might not have otherwise been as apparent.

\section{References}

1. Chorin, A. J. "A Numerical Method for Solving Incompressible Viscous Flow Problems," Journal of Computational Physics, Vol. 2, 1967, pp. 12-26.

2. Turkel, E., Fiterman, A., and van Leer, B. "Preconditioning and the Limit to the Incompressible Flow Equations," AIAA Computational Fluid Dynamics Conference, AIAA 87-1107-CP, 1987.

3. Turkel, E., "Preconditioning Methods for Solving the Incompressible and Low Speed Compressible Equations," Journal of Computational Physics, Vol. 72, 1987, pp. 277-298.
4. Turkel, E., "A Review of Preconditioning Methods for Fluid Dynamics," Applied Numerical Mathematics, Vol. 12, 1993, pp. 257-284.

5. Choi, Y.-H. and Merkle, C. L., "The Application of Preconditioning to Viscous Flows," Journal of Computational Physics, Vol. 105, 1993, pp. 207-223.

6. Radespiel, R., Turkel, E., and Kroll, N. "Assessment of Preconditioning Methods," DLR Report, 1995.

7. Radespiel, R. and Turkel, E. "Assessment of Preconditioning Methods," Proceedings of Cerca Conference, 1995.

8. Darmofal, D. L. and Schmid, P. J. "The Importance of Eigenvectors for Local Preconditioners of the Euler Equations," 12th AIAA Computational Fluid Dynamics Conference, 1995, pp. 102-117. 
9. Fiterman, A., Turkel, E., and Vatsa, V. "Pressure Updating Methods for the Steady-State Fluid Equations," Proceedings 12th AIAA Computational Fluid Dynamics Conference, AIAA Paper 95-1652, 1995.

10. Turkel, E., Vatsa, V. N., and Radespiel, R. "Preconditioning Methods for Low-Speed Flows," 14th AIAA Applied Aerodynamics Conference, AIAA Paper 96-2460-CP, 1996.

11. Chima, R. V., and Yokota, J. W., "Numerical Analysis of Three-Dimensional Viscous Internal Flows," AIAA Journal, Vol. 28, No. 5, May 1990, pp. 798806 (also NASA TM-100878).

12. Chima, R. V., "Viscous Three-Dimensional Calculations of Transonic Fan Performance," in CFD Techniques for Propulsion Applications, AGARD Conference Proceedings No. CP-510, AGARD, Neuilly-Sur-Seine, France, Feb. 1992, pp. 21-1 to 21-19 (also NASA TM-103800).

13. Baldwin, B.S., and Lomax, H., "Thin-Layer Approximation and Algebraic Model for Separated Turbulent Flows," AIAA Paper 78-257, Jan. 1978.

14. Giles, M. B. "Nonreflecting Boundary Conditions for Euler Equation Calculations," AIAA Journal, Vol. 28, No. 12, Dec. 1990, pp. 2050-2058.

15. Jameson, A., Schmidt, W., and Turkel, E., "Numerical Solutions of the Euler Equations by Finite Volume Methods Using Runge-Kutta Time-Stepping Schemes," AIAA Paper 81-1259, June 1981.

16. Arnone, A., "Viscous Analysis of Three-Dimensional Rotor Flows Using a Multigrid Method," NASA TM-106266 (ICOMP-93-25), July 1993.

17. Jameson, A., and Baker, T. J., "Solution of the Euler Equations for Complex Configurations," AIAA Paper 83-1929, July 1983.

18. Martinelli, L., and Jameson, A., "Validation of a Multigrid Method for the Reynolds Averaged Equations," AIAA Paper 88-0414, Jan. 1988.

19. Kunz, R. F., and Lakshminarayana, B., "Computation of Supersonic and Low Subsonic Cascade Flows Using an Explicit Navier-Stokes Technique and the $\mathrm{K}-\varepsilon$ Turbulence Model," in Computational Fluid Dynamics Symposium on Aeropropulsion, NASA CP-10045, Apr. 1990.

20. Jameson, A. "Analysis and Design of Numerical Schemes for Gas Dynamics 1, Artificial Diffusion, Upwind Biasing, Limiters and Their Effect on Accuracy and Multigrid Convergence," International Journal of Computational Fluid Dynamics, Vol. 4, 1995, pp. 171-218 (also NASA CR-196447, Aug. 1994).
21. Tatsumi, S., Martinelli, L., and Jameson, A. "Design, Implementation and Validation of Flux Limited Schemes for the Solution of the Compressible Navier-Stokes Equations," AIAA Paper 940647 , Jan. 1994.

22. Tatsumi, S., Martinelli, L., and Jameson, A. "New High Resolution Scheme for Compressible Viscous Flow with Shocks," AIAA Paper 95-0466, Jan. 1995.

23. Swanson, R. C., Radespiel, R., and Turkel, E. "Artificial Dissipation Models and Limiters," AIAA Paper 97-1945, June 1997.

24. van Leer, B. "Towards the Ultimate Conservative Difference Scheme. V: A Second Order Sequel to Godunov's Method," Journal of Computational Physics, Vol. 32, 1979, pp. 101-136.

25. Tweedt, D. L., and Chima, R. V. "Rapid Numerical Simulation of Viscous Axisymmetric Flow Fields," AIAA Paper 96-0449 (also NASA TM-107103), Jan. 1996.

26. Jiang, Y. T., Damodaran, M., and Lee, K. H."High Resolution Finite Volume Calculation of Turbulent Transonic Flow Past Airfoils," AIAA Paper 962377-CP, 1996.

27. Pulliam, T. H., and Steger, J. L., "Implicit FiniteDifference Simulations of Three-Dimensional Compressible Flow," AIAA Journal, Vol. 18, No. 2, Feb. 1980, pp. 159-167.

28. Cooper, G. K., and Sirbaugh, J. R. "PARC Code: Theory and Usage," AEDC-TR-89-15, Dec. 1989. pp. 36-37.

29. Wood, J. R., Adam, P. W., and Buggele, A. E. "NASA Low-Speed Centrifugal Compressor for Fundamental Research," NASA TM-83398, 1983.

30. Hathaway, M. D., Wood, J. R., and Wasserbauer, C. A. "NASA Low-Speed Centrifugal Compressor for Three-Dimensional Viscous Code Assessment and Fundamental Flow Physics Research," Journal of Turbomachinery, Vol. 114, Apr. 1992, pp. 295-303.

31. Hathaway, M. D., Chriss, R. M., Strazisar. A. J., and Wood, J. R. "Laser Anemometer Measurements of the Three-Dimensional Rotor Flow Ficld in the NASA Low-Speed Centrifugal Compressor," NASA TP-3527 (ARL-TR-333), June 1995.

32. Hathaway, M. D., and Wood, J. R. "Application of a Multi-Block CFD Code to Investigate the Impact of Geometry Modeling on Centrifugal Compressor Flow Field Predictions," ASME Paper 96-GT-372 (also NASA TM-107198), June 1996. 
Public reporting burden for this collection of information is estimated to average 1 hour per response, including the time tor reviewing instructions, searching existing data sources, gathering and maintaining the data needed, and completing and reviewing the collection of information. Send comments regarding this burden estimate or any other aspect of this collection of information, including suggestions for reducing this burden, to Washington Headquarters Services, Directorate for information Operations and Reports, 1215 Jefferson Davis Highway, Suite 1204, Arlington, VA 22202-4302, and to the Office of Management and Budget, Paperwork Reduction Pro;ect (0704-0188), Washington, DC 20503.
1. AGENCY USE ONLY (Leave blank)
2. REPORT DATE
October 1997
3. REPORT TYPE AND DATES COVERED
Technical Memorandum

\section{TITLE AND SUBTITLE}

5. FUNDING NUMBERS

Preconditioning for Numerical Simulation of Low Mach Number

Three-Dimensional Viscous Turbomachinery Flows

6. AUTHOR(S)

Daniel L. Tweedt, Rodrick V. Chima, and Eli Turkel

7. PERforming ORganization name(S) AND ADDRESS(ES)

8. Performing organization REPORT NUMBER

National Aeronautics and Space Administration

Lewis Research Center

Cleveland, Ohio 44135-3191

E-10879

9. SPONSORINGMONITORING AGENCY NAME(S) AND ADDRESS(ES)

10. SPONSORINGMONITORING AGENCY REPORT NUMBER

National Aeronautics and Space Administration

Washington, DC 20546-0001

NASA TM-113120

ICOMP-97-11

\section{SUPPLEMENTARY NOTES}

Prepared for the 28th Fluid Dynamics Conference sponsored by the American Institute of Aeronautics and Astronautics, Snowmass, Colorado, June 29-July 2, 1997. Daniel L. Tweedt and Rodrick V. Chima, NASA Lewis Research Center; Eli Turkel, Tel-Aviv University, School of Mathematical Sciences, Tel-Aviv, Israel and Institute for Computational Mechanics in Propulsion. Responsible person, Daniel L. Tweedt, organization code 5810, (216) 433-3592.

12a. DISTRIBUTION/AVAILABILITY STATEMENT

Unclassified - Unlimited

Subject Category 07

This publication is available from the NASA Center for AeroSpace Information, (301) 621-0390.

13. ABSTRACT (Maximum 200 words)

A preconditioning scheme has been implemented into a three-dimensional viscous computational fluid dynamics code for turbomachine blade rows. The preconditioning allows the code, originally developed for simulating compressible flow fields, to be applied to nearly-incompressible, low Mach number flows. A brief description is given of the compressible Navier-Stokes equations for a rotating coordinate system, along with the preconditioning method employed. Details about the conservative formulation of artificial dissipation are provided, and different artificial dissipation schemes are discussed and compared. The preconditioned code was applied to a well-documented case involving the NASA large low-speed centrifugal compressor for which detailed experimental data are available for comparison. Performance and flow field data are compared for the near-design operating point of the compressor, with generally good agreement between computation and experiment. Further, significant differences between computational results for the different numerical implementations, revealing different levels of solution accuracy, are discussed.

14. SUBJECT TERMS

Preconditioning; Computational fluid dynamics; CFD; Turbomachinery

17. SECURTYY CLASSIFICATION OF REPORT Unclassified

18. SECURITY CLASSIFICATION
OF THIS PAGE
Unclassified

Unclassified
19. SECURITY CLASSIFICATION OF ABSTRACT

20. LIMITATION OF ABSTRACT 